
$\$$ Research Square
Preprints are preliminary reports that have not undergone peer review.
They should not be considered conclusive, used to inform clinical practice, or referenced by the media as validated information.

\title{
Bionanosystem Based on Platycodon Grandifloras "Guides the Medicine Upward" for Targeting Lung Inflammation to Calm the Cytokine Storm
}

Yi Li

Yantai University

Chunjing Guo

Ocean University of China

Qiang Chen

Yantai University

Yanguo Su

Yantai University

Huimin Guo

Yantai University

Daquan Chen ( $\nabla$ cdq1981@126.com )

Yantai University https://orcid.org/0000-0002-6796-0204

\section{Research Article}

Keywords: Acute lung injury, Platycodon grandiflorum polysaccharide, Biomimetic, Guide theory, Cytokine storms

Posted Date: August 19th, 2021

DOI: https://doi.org/10.21203/rs.3.rs-793730/v1

License: (9) This work is licensed under a Creative Commons Attribution 4.0 International License. Read Full License 


\section{Abstract}

Pneumonia can lead to high morbidity and mortality due to uncontrolled inflammation of the lung tissue. More and more evidences indicate that cytokine storms play a vital role in the progression of inflammatory lung diseases. Blocking cytokine storms may be one of the keys to saving the life of patients with severe pneumonia. According to the guide theory of Traditional Chinese Medicine (TCM) and the inherent affinity of macrophages for the site of inflammation, we constructed a bionic drug delivery system (MPNPs) derived from macrophage-membrane (MM) encapsulated ROS-responsive Platycodon grandiflorum polysaccharide (PGP) nanoparticles (PNPs) to calm down the cytokine storm and improve lung inflammation. By loading the anti-inflammatory agent Curcumin (Cur), we could clearly find that MPNPs@Cur were better targeted to the inflammatory cell and more significantly suppressed the release of proinflammatory factors such as IL- 6 and TNF-a. In acute lung injury (ALI) mice treated with MPNPs@Cur significantly reduced inflammation and cytokine storm syndrome, while also alleviating symptoms in mice with ALI. Interestingly, we found that the PNPs also had potent pulmonary targeting compared to other polysaccharide carriers, which is in line with the guide theory of the attribution of the Platycodon grandiflorum to the lung. Additionally, MM segregate pro-inflammatory cytokines to suppress local inflammation. The synergistic effect of drug therapy and the separation of inflammatory cytokines from this biomimetic drug delivery system leads to an increase in the therapeutic effect of ALI. Rational design of drug delivery platform to improve the treatment of lung injury is revealed in our study, which inherits and develops the important theories of TCM through the perfect combination of guide theory and modern biomimetic nanotechnology, as well as provides experimental scientific basis for the clinical application of channel ushering drugs.

\section{Introduction}

Inflammation is a defense mechanism that protects body from damage caused by infections and tissue injury. However, uncontrolled hyperinflammation can also induce an overwhelming local/systemic inflammatory response leading to life-threatening diseases such as pneumonia, acute lung injury (ALI), which can rapidly cause acute respiratory distress syndrome (ARDS)[1-4]. For example, the novel coronavirus disease 2019 (COVID-19), which is spreading worldwide, has infected more than 170 million people and killed more than 3.5 million of people in worldwide so far. Based on current clinical studies, sudden exacerbations in patients with their infections have been found to be closely associated with cytokine storm, which mark an uncontrolled and dysfunctional immune response that will likely lead to serious consequences and even death[5-8].

More and more evidences indicate that suppression of cytokine storm may be o one key to save the life of patients with severe pneumonia[9, 10]. Currently, glucocorticoids are used in the treatment of heavy and critical cases in the existing protocols to exert immunosuppressive effects and to control cytokine storm[11, 12]. Although promising, frequent long-term use or high doses of glucocorticoids may lead to the risk of serious complications and sequelae, such as osteonecrosis and hyperglycemia[13-15]. In addition, there is a demand for further development of microenvironment-responsive targeted drug 
delivery systems to modulate the local inflammatory immune response to improve the inflammatory microenvironment (IME) at the lesions considering the unique characteristics of the IME[16-18], including low $\mathrm{pH}$ and overproduction of reactive oxygen species (ROS), thus improving the therapeutic efficacy as well as reducing the toxic side effects.

In light of the Chinese Herbal Formula played a significant role in this control and treatment of this epidemic, for example, Qingfei Paidu Decoction has become an important tool in the treatment of COVID$19[19,20]$. It was found that in the face of complex pathogenesis of cytokine storm, Traditional Chinese Medicine (TCM) with multiple targets can be more advantageous than chemotherapeutics with clear targets through holistic modulation[21-23]. Curcumin (Cur), a natural plant extract, has been shown to inhibit viral infections, reduce the severity of lung injury and inhibit subsequent fibrosis by calming down cytokine storm, thereby increasing survival rates and improving symptoms in mice with pneumonia[24]. Besides, Platycodon grandiflorum is mostly used as a channel ushering drug in classical formulas, acting as an "oar" to guide the upward movement of medicine. For instance, in Medical Heart Enlightenment, there is a formula that combines Platycodon grandiflorum with Curcuma to guide the upward movement of medicine to treat hypochondriac pain. Inspired by the guide theory of TCM and modern targeting drug delivery system, we can construct or modify the drug delivery system by the effective components of the channel ushering drug to achieve the targeting purpose, such as liver targeting based on the Angelica polysaccharide (AP) and establishing bionic vector for the treatment of liver cancer[25]. Platycodon grandiflorum polysaccharide (PGP), as the active ingredient of Platycodon grandiflorum, has been studied mostly for its pharmacological effects such as antitumor, anti-inflammatory and antioxidant[26, 27], while the study as a drug delivery vehicle is currently a complete blank. Inspired by the hepatic targeting ability of AP and the unique IME, ROS-responsive strategies based on PGP can be developed for drug delivery in lung diseases.

Cellular delivery systems are received mounting interest for their excellent biocompatibility and exceptional drug delivery behavior[28-31]. Macrophages as one of inherent cells in the body, which can act as drug delivery systems are equipped with several advantages compared with other synthetic delivery systems[32]. For instance, as a role of immune cells, macrophages have an intrinsic affinity for sites of inflammation, and chemokines on the surface of macrophage membranes (MM) such as chemokine receptors CCR2, CCR6 and CXCR1 can trigger macrophages to adhere to inflammatory or tumor sites[33,34]. Simon et al[35]. recently revealed that MM-encapsulated drug delivery methods may be more suitable than live cell methods for the treatment of inflammatory diseases, demonstrating the broad promise of cell membrane-coated nanoparticles. Wang et al[36]. utilized MM-encapsulated nanoparticles loaded with rapamycin for targeted treatment of atherosclerosis and exhibited outstanding efficacy. Zhang et al[37]. reported a multi-antigen nano-toxin vaccine based on MM-encapsulated nanoparticles to combat inflammatory lung infections. There is a growing amount of evidence that MMcoated drug delivery systems may be a powerful platform for targeted inflammatory therapies.

In this study, given the important role of macrophages in the inflammatory process, we attempted to structure dual targeting biomimetic drug delivery system (MNPs) with MM coating for targeted therapy of 
pneumonia (Scheme 1). The core is a ROS-responsive nanoparticle loaded with Cur (PNPs@Cur) constructed on the basis of PGP, which achieves precise drug release at the site of inflammation (Scheme 2). Interestingly, we discovered that PGP nanoparticles (PNPs) were also efficiently aggregated in the lungs of model mice compared to the control group. In mice models of $A L I$, we systematically evaluated the targeting efficiency and therapeutic efficacy of PNPs@Cur and MM coated PNPs@Cur (MNPs@Cur), significantly reducing inflammation and cytokine storm syndrome and alleviating symptoms in mice with ALI compared to the free drug group. We hypothesized that MNPs@Cur, which possess the dual targeting of TCM channel ushering and macrophage intrinsic chemotaxis, could more effectively accumulate in the damaged lung and thus effectively inhibit the progression of lung disease.

\section{Material And Methods}

\section{Materials}

4-hydroxymethyl phenylboronic acid pinacol ester (4-PBAP) and N, N'-carbonyl diimidazole (CDI) were purchased from Aladdin (China). Platycodon grandiflorum polysaccharides were supplied by Shanghai Winherb Medical Technology Co., Ltd. 1,1-dioctadecyl-3,3,3,3-tetramethylindotricarbocyaine iodide (DiR) was obtained from Beijing Bioss Biotechnology Co. DAPI, Cell Total Protein Extraction kits, Bicinchoninic Acid Assay (BCA) protein assay kit and Membrane Protein Extraction kits were supplied by Beyotime Institute of Biotechnology (China). TNF-a and IL-6 ELISA kit were purchased from Beijing Solarbio Technology Co., Ltd. (China). Antibody CCR2 (anti-rabbit, \#ABP53395) special for mouse were purchased from Abbkine (China), with a dilution ratio of 1:1000 for antibody. Antibodies $\beta$-Actin (\#4967S) and HRPconjugated anti-rabbit IgG (\#7074) were purchased from Cell Signaling Technology (USA), with a dilution ratio of 1:1000 for both antibodies during experimental use. All other chemical reagents were supplied by Aladdin (China). All biological reagents, and other cell culture medium were used as received following our previous protocols.

\section{Synthesis and characterization of copolymers.}

The synthesis process was carried out according to the relevant literature[35, 38]. Briefly, the ROS reactive groups were first synthesized. The 4-hydroxymethylbenzeneboronic acid pinacol ester (4-PBAP, $5 \mathrm{mmol}$ ) and $\mathrm{N}, \mathrm{N}^{\prime}$-carbonyl diimidazole (CDI, $10 \mathrm{mmol}$ ) were added to the reaction flask. After added $30 \mathrm{~mL}$ of anhydrous dichloromethane to fully dissolve the reaction and stirred at room temperature for $12 \mathrm{~h}$, the unreacted raw material was washed with pure water $(3 \times 25 \mathrm{~mL})$ followed by saturated $\mathrm{NaCl}(3 \times 20 \mathrm{~mL})$, discarded the aqueous layer and dried overnight under anhydrous $\mathrm{Na}_{2} \mathrm{SO}_{4}$. The ROS-responsive groups CDI-PBAP were obtained by reduced-pressure drying, and their chemical structures were characterized by NMR as well as IR spectroscopy.

To prepare modified Platycodon grandiflorum polysaccharides (Oxi-PGP), we performed structural modification of PGP by prepared CDI-PBAP, namely PGP $(5 \mathrm{mmol})$ dissolved in formamide $(4 \mathrm{~mL})$, added DMAP (10 mmol) dissolved in anhydrous dimethyl sulfoxide (DMSO), and finally added CDI-PBAP (10 
$\mathrm{mmol}$ ), and stirred the mixture overnight at room temperature with a magnetic stirrer. Subsequently, the resulting solution was dialyzed in deionized water and centrifuged at $1500 \mathrm{rpm}$ for $5 \mathrm{~min}$ to obtain the supernatant, which was freeze-dried and prepared for use, and the successful synthesis of the modified product Oxi-PGP was verified by a series of characterizations.

\section{Preparation and characterization of ROS responsive PNPS and PNPs@Cur}

ROS-responsive nanoparticles were similarly prepared by dialysis. Oxi-PGP $(10 \mathrm{mg})$ was weighed and dissolved in anhydrous DMSO $(5 \mathrm{~mL})$, and the resulting solution was dialyzed in deionized water (1000 $\mathrm{mL}$ ) (MW: 8000-14000 Da) for $36 \mathrm{~h}$, with water replacement at $4 \mathrm{~h}$ intervals. The final dialyzed solution was passed through the syringe filter (pore size $0.45 \mu \mathrm{m}$ ) to remove insoluble material to obtain the purified PNPs solution. To validate the nanoparticles for ROS responsiveness, the PNPs were incubated in phosphate-buffered saline (PBS) containing different concentrations of $\mathrm{H}_{2} \mathrm{O}_{2}(0,0.1,0.25,0.5$ and $1 \mathrm{mM})$ for $6 \mathrm{~h}$. At predetermined time points, the transmittance at $500 \mathrm{~nm}$ was measured separately by UV spectrophotometer and the respective percentage of hydrolysis was calculated.

For preparation of PNPs@Cur, we dissolved the lyophilized carrier material with Cur in anhydrous DMSO at a ratio of $6: 1(\mathrm{w} / \mathrm{w})$. The solution was dialyzed in pure water (MW: 8000-14000 Da) to remove the free drug and organic solvents. A portion of the resulting PNPs@Cur solution was taken and stored at $4^{\circ} \mathrm{C}$, and the rest was freeze-dried for subsequent experiments. Subsequently, particle size, polydispersity index (PDI) and zeta potential were determined by dynamic light scattering (DLS) with a Delsa Nano C (Beckman Coulter, USA), morphology and size were determined by transmission electron microscopy (TEM, JEM-1400 Plus). Chitosan-based drug delivery nanosystems (CNPs@Cur) were prepared in a similar manner to the above process.

\section{Drug encapsulation and release in vitro}

The drug content in PNPs@Cur was measured by HPLC (Agilent 1260, USA)). The measurement was performed using $0.5 \%$ acetic acid aqueous solution and acetonitrile $(35: 65, \mathrm{~V} \%)$ as the mobile phase with a detection wavelength of $425 \mathrm{~nm}$. Then, the loading content (LC, \%) and encapsulation efficiency (EE, \%) are calculated according to the following equations 1 and 2, respectively.

$$
\begin{aligned}
& L C(\%)=\frac{\text { weight of loaded drugs }}{\text { weight of drug loaded NPs }} \times 100 \% \\
& E E(\%)=\frac{\text { weight of loaded drug }}{\text { weight of drug in feed }} \times 100 \%
\end{aligned}
$$

The release behavior of Cur in vitro was investigated using dialysis method. For the examination of the release of PNPs@Cur in response to ROS, PNPs@Cur $(5 \mathrm{mg})$ dissolved in deionized water $(4 \mathrm{~mL})$ were placed in dialysis bags in PBS $(40 \mathrm{~mL})$ containing different concentrations of $\mathrm{H}_{2} \mathrm{O}_{2}(0,0.5$ and $1 \mathrm{mM})$. At predetermined time points, $1 \mathrm{~mL}$ of the medium was taken separately for HPLC analysis and $1 \mathrm{~mL}$ of 
fresh medium was reinjected into the corresponding concentration of PBS. The cumulative release rate of Cur was calculated using the following Eq. 3.

$$
\text { Cumulative drug release }(\%)=\frac{M_{t}}{M_{0}} \times 100 \%
$$

where $M_{t}$ is the weight of drug released at time $t$ and $M_{0}$ is the weight of loaded Cur. The release of the experiments was performed in triplicate.

\section{Preparation of the MM}

By utilizing the Membrane Protein Extraction Kit[36, 39], we obtained purer cells membrane materials. Briefly, the collected RAW264.7 cells were resuspended in membrane protein extraction buffer for 10 min under ice bath conditions, followed by transferring the cell suspension to a glass homogenizer and homogenizing about 20 times to ensure cell fragmentation. After centrifugation at $2000 \times \mathrm{g}$ and $4^{\circ} \mathrm{C}$ for about $10 \mathrm{~min}$, the supernatant was further centrifuged at $14000 \times \mathrm{g}$ for about $30 \mathrm{~min}$ to collect the cell membrane precipitate and repeated twice to obtain purified cell membranes. Finally, the total protein content in the purified MM was analyzed by the bicinchoninic acid assay (BCA) protein assay. The prepared membrane material was stored at $-80^{\circ} \mathrm{C}$ for future studies.

\section{Preparation of characterization MNPs and MNPs@Cur}

MNPs and MNPs@Cur were prepared by membrane extrusion. In brief, MM vesicles were mixed with PNPs and PNPs@Cur (1:5, w/w), followed by substantial sonication for 2 min using a bath sonicator at $40 \mathrm{kHz}$ and $100 \mathrm{~W}$ power. Finally, nanoparticles with MM coating were obtained by repeated extrusion through 400, $200 \mathrm{~nm}$ polycarbonate porous membranes sequentially for at least 30 times by a microextruder (LiposoEasy LE-1, USA).

Then, particle size, PDI and zeta potential were also measured by DLS, as well as morphology by TEM. For further validation of the successful coating of cell membranes, the particular membrane proteins on the surface of macrophages were characterized by Western blotting. Total protein was extracted from macrophages, MM and MNPs by using a protein extraction kit, respectively, and protein concentration was determined by BCA protein assay. Approximately $25 \mu \mathrm{g}$ of protein was subsequently added to protein loading buffer, boiled for $10 \mathrm{~min}$, and then electrophoresed on a $10 \%$ sodium dodecyl sulfate polyacrylamide gel and transferred to PVDF membranes. Primary and secondary antibodies were added sequentially after closure with $5 \%$ skim milk powder, incubated for $2 \mathrm{~h}$ at room temperature, and specific bands were observed with an enhanced chemiluminescence detection kit.

\section{Cytotoxicity and hemolysis assay}

The cytotoxicity of PNPs and MNPs were investigated by incubation in RAW264.7 and HUVECs (human umbilical vein endothelial cells) cells. Simply, cell densities of approximately 8000 cells per well were inoculated in 96-well plates, respectively, and after overnight incubation, the medium was replaced with fresh medium containing different concentrations of PNPs or MNPs. After continued incubation for $48 \mathrm{~h}$ at $37^{\circ} \mathrm{C}$ in a cell incubator containing $5 \% \mathrm{CO}_{2}, \mathrm{MTT}(5 \mathrm{mg} / \mathrm{mL})$ reagent was added and incubated for 
another $4 \mathrm{~h}$. Then, the supernatant was removed and DMSO was added to dissolve the bottom residue. The absorbance of each solution was read by multi-wall plate reader at $570 \mathrm{~nm}$ to obtain the corresponding cell viability.

As previously reported, direct contact method was used to detect the hemolytic properties of PNPs and MNPs in vitro. Briefly, $2 \%$ erythrocyte suspensions were prepared by taking blood from the orbits of mice. Then, $0.5 \mathrm{~mL}$ of the red cell suspension was mixed well with saline, purified water, PNPs and MNPs solutions $(0.5 \mathrm{~mL}, 2 \mathrm{mg} / \mathrm{mL})$, respectively. These specimens were then incubated continuously at $37^{\circ} \mathrm{C}$ for 2 hours. The absorbance of the supernatant was measured at $570 \mathrm{~nm}$ using a microplate reader to determine the hemoglobin released from the lysed erythrocytes. Saline and pure water were used as negative and positive controls, respectively.

\section{In vitro binding and intracellular drug release}

The targeting ability of MNPs was further investigated employing in vitro cellular uptake assays. Cellular escape ability was studied first in normal macrophages. An appropriate number of cells were seeded in 12-well plates, cultured overnight, and the original medium was replaced with fresh medium containing free Cur, PNPs@Cur, and MNPs@Cur. After incubation for 1-4 hours, respectively, the cells were washed three times with PBS, then fixed with $4 \%$ paraformaldehyde for 15 minutes, then washed three times with PBS, and finally stained with DAPI for 10 minutes and washed three times before observation by microscopy cell uptake. To imitate the inflammatory cell model, macrophages as well as HUVECs were activated with lipopolysaccharide (LPS, $100 \mathrm{ng} / \mathrm{mL}$ ), respectively, and the subsequent steps were performed as described above to observe the targeting ability of MNPs@Cur in inflammatory cells. Likewise, in order to verify the potential role of PGP as priming components of Platycodon grandiflorum, the ability of PNPs@Cur and CNPs@Cur was compared respectively in terms of their uptake by macrophages in different states.

To explore further the intracellular drug release behavior, another fluorescent probe, NR, was selected to be loaded into the nanoparticles. NR did not fluoresce on its own, but excited intense fluorescence when it came into contact with intracellular lipids. The activated macrophages were obtained by LPS pretreatment, and the uptake was observed by replacing the original medium with medium containing PNPs@NR and MNPs@NR after 1, 2, 4 and 8 hours of continuous incubation.

\section{Animals}

Female BALB/c mice (6-8 weeks old, 16-18 g) were purchased from Jinan Pengyue Laboratory Animal Breeding Co. All mice were acclimatized to their environment for at least 7 days prior to the experiments. A protocol for animal use was followed by the China Animal Protection Commission and Yantai University for all animal-related procedures. All experiments on animals complied with ethical guidelines.

\section{Animal model induction and treatment}

Acute lung injury (ALI) mouse models were established according to the relevant literature. Mice were injected intraperitoneally with LPS $(10 \mathrm{mg} / \mathrm{kg})$, and after $5 \mathrm{~h}$ of successful modeling, randomly grouped 
$(\mathrm{n}=3)$ and treated with intravenous saline, free Cur, PNPs@Cur and MNPs@Cur, respectively, and euthanized at $24 \mathrm{~h}$ after treatment for subsequent experimental analysis.

\section{In vivo targeting to inflammatory site}

After establishment of ALI mice, the mice were randomly grouped and injected intravenously with fluorescent dyes DiR (1,1-dioctadecyl-3,3,3,3-tetramethylindotricarbocyaine iodide),CNPs@DiR, PNPs@DiR andMNPs@DiR, respectively, and normal mice were used as the control group, and the fluorescence distribution of organs in each group was observed by imaging the collected organs at predetermined times with the in vivo imaging system (IVIS).

\section{Cytokine assay}

The levels of cytokines (IL-6 and TNF- $\alpha$ ) in cell supernatants and serum samples of different treatment groups were measured according to the ELISA KIT instruction manual.

\section{Evaluation of oxidative stress in lung tissue}

The lung tissues were collected from different treatment groups for weighing and then $10 \%$ lung tissue homogenates were prepared. The levels of MPO and MDA were measured according to the procedure of the relevant kit.

\section{Lung wet/dry ratios}

The lung tissues from the mice in the different treatment groups were removed and weighed immediately to obtain wet weights, followed by drying the tissue samples in an oven at $50^{\circ} \mathrm{C}$ to a constant weight. Finally, the degree of pulmonary edema was quantified by the ratio of wet weight to dry weight.

\section{Histopathological analysis}

For histological analysis, major organs (heart, liver, spleen, lung and kidney) were collected after different treatments, fixed overnight in $4 \%$ paraformaldehyde and paraffin embedded, then sectioned and stained with hematoxylin-eosin (H\&E) for tissue evaluation.

\section{Statistical analysis}

The results obtained are expressed as mean $\pm S D$, all statistical analyses were acquired by using GraphPad Prism version 8.0 software (GraphPad, USA). One-way ANOVA and two-way ANOVA were utilized for statistical analysis. Significance levels were set at $* P<0.05, \star \star P<0.01$ and ${ }^{*} * P<0.001$. Expression of all fluorescence intensities in the experiments were further calculated by Image $\mathrm{J}$ software.

\section{Results And Discussion}

\section{Synthesis and characterization of PNPs and MNPs}


Firstly, we obtained the amphiphilic carrier material Oxi-PGP by structural modification of PGP, followed by self-assembly to obtain nanoparticles (PNPS) with ROS responsiveness. The synthesis process of the carrier materials is shown in Fig. 1a. The successful construction of Oxi-PGP was verified by ${ }^{1} \mathrm{H}$ NMR (Fig. 1b), the peaks appearing at 3.0-4.0 ppm and 7.0-8.2 ppm belonged to PGP as well as ROSresponsive groups, respectively. The marked decrease in the absorption peak of hydroxyl group at 3300 $\mathrm{cm}^{-1}$ and the appearance of carbonyl and phenyl characteristic peaks at $1748 \mathrm{~cm}^{-1}$ and $1650-1500$ $\mathrm{cm}^{-1}$ in Fourier transform infrared spectroscopy (FT-IR) further confirmed the successful preparation of the carrier material (Fig. 1c). Subsequently, the drug encapsulation efficiency (EE) and drug loading efficiency (DLC) of PNPs@Cur were 76.6\% and 11.3\%, respectively, obtained by HPLC, indicating the successful drug encapsulation. Dynamic light scattering (DLS) analysis showed that the hydrodynamic diameter of PNPs was about $91.1 \mathrm{~nm}$, the PDI was 0.139 , and the zeta potential of PNPs was $-23.29 \mathrm{mV}$ (Fig. 2a). Transmission electron microscopy (TEM) indicated that these PNPs particles had a spherical structure with a particle size of about $80 \mathrm{~nm}$ (Fig. 2d).

The ROS-responsive drug delivery system constructed in this study, as its hydrophobic core phenylborate polymer could be degraded under $\mathrm{H}_{2} \mathrm{O}_{2}$ conditions, disrupted the nanoparticle structure allowing precise drug release in the inflammatory microenvironment. To investigate the ROS responsiveness of PNPs, we selected phosphate-buffered saline (PBS) containing different concentrations of $\mathrm{H}_{2} \mathrm{O}_{2}$ to measure the degree of hydrolysis of nanoparticles at predetermined time points, namely the change in transmittance at $500 \mathrm{~nm}$. The $\mathrm{H}_{2} \mathrm{O}_{2}$ concentrations used in this study were based on previous simulated in vitro inflammatory lesion tissue $\mathrm{H}_{2} \mathrm{O}_{2}$ levels[40]. As illustrated in Fig. 2c, the degree of hydrolysis of PNPsd gradually increased with increasing $\mathrm{H}_{2} \mathrm{O}_{2}$ concentration, and the particle solution was almost completely transparent within $3 \mathrm{~h}$ at $1.00 \mathrm{mM}$ concentration. In contrast, the particles in PBS exhibited only a moderate decrease in absorbance within $6 \mathrm{~h}$ due to the partial precipitation of PNPs. With the gradual structural disruption of the nanoparticles, the release of the drug should be increased. This speculation was subsequently confirmed by our in vitro cumulative release profiles at different $\mathrm{H}_{2} \mathrm{O}_{2}$ concentrations (Fig. 2f). At a concentration of $1.00 \mathrm{mM} \mathrm{H}_{2} \mathrm{O}_{2}$, PNPs@Cur exhibited a cumulative drug release rate of up to $76 \%$, however, only about $20 \%$ of the drug was released in the absence of peroxide treatment. Collectively we have successfully constructed ROS-responsive drug delivery systems.

In order to acquire MNPs, the macrophage membranes were first oscillatory fused with PNPs in ultrasound and then passed through 400 and $200 \mathrm{~nm}$ polycarbonate porous membranes by extrusion, respectively. Compared with the uncoated PNPs, DLS (Fig. 2b) indicated that the MNPs hydrodynamic diameter increased from $91.1 \mathrm{~nm}$ to $125.3 \mathrm{~nm}$ with a more negative zeta potential, which was closer to the zeta potential of the macrophage surface (Fig. $2 \mathrm{~g}$, h). Within the TEM images MNPs exhibited a spherical core-shell structure (Fig. 2e), each nanoparticle was encapsulated by a single layer of cell membrane, and the coating thickness of the nanoparticles was about $9 \mathrm{~nm}$, consistent with the thickness of the cell membrane, suggesting a successful wrap of the cell membrane. Furthermore, the presence of the key membrane antigen CCR2 (receptor for monocyte chemoattractant protein-1 (MCP-1)), which tends immune cells to the site of inflammation, was detected by Western blot on the surfaces of macrophages, 
macrophage membranes, and MNP. In contrast, $\beta$-Actin was largely absent compared to macrophages, demonstrating the higher purity of macrophage membrane extraction. However, no protein signal was detected from PNPs, further indicating that macrophage membranes were successfully coated and maintained on the surface of MNPs (Fig.3b-d).PNPs@Cur and MNPs@Cur both exhibited relatively stable sizes in PBS containing 10\% fetal bovine serum (FBS) after long-term storage at room temperature, indicating satisfactory stability (Fig. 3a). We also made an examination of the in vitro release of MNPs@Cur, the release rate of MNPs@Cur was quite similar to that of PNPs@Cur under the same conditions (Fig. 2i), indicating that the macrophage membrane coating had no significant effect on the responsiveness of PNPs to the inflammatory microenvironment.

\section{In vitro binding to the site of inflammation}

Whether the drug delivery system could be uptaken by target cells is a prerequisite for maximizing drug efficacy and reducing side effects on other tissues and organs. Therefore, it is an essential indicator to avoid phagocytosis by macrophages of the reticuloendothelial system to verify the drug delivery system. There is increasing evidence that MM-encapsulated nanoparticles can prevent the phagocytosis of macrophages[39, 41]. As the model drug Cur itself is fluorescent, the uptake efficiency of MNPs@Cur, PNPs@Cur was evaluated in RAW264.7 cells, respectively, with the free drug as a control group. We could find a time-dependent uptake in each group (Fig. 4a), but intense green fluorescence could be observed in macrophages incubated with free Cur for $4 \mathrm{~h}$, apparently indicating that the free drug was taken up by macrophages. In contrast, little fluorescence was observed in macrophages cultured with MNPs@Cur, implying that the drug delivery system wrapped by the macrophage membrane may be an effective strategy to evade macrophage clearance. Interestingly, we found that less fluorescence was also present in the PNPs@Cur incubation group. To further investigate the uptake mechanism,CNPs@Cur was used for comparison, and PNPs@Cur could also be effective for cellular escape (Fig. 5a), implicating that PNPs@Cur may also have better drug delivery properties.

Subsequently, we validated whether MNPs@Cur can effectively target damaged inflammatory cells. The conversion of RAW264.7 cells into activated macrophages was induced by LPS thus to simulate the establishment of a cellular inflammation model[42, 43]. It was evident that intense fluorescence could be observed in MNPs@Cur incubation (Fig. 4b), indicating a significant uptake by inflammatory macrophages, implying no discrimination against macrophage-membrane coated nanoparticles. To further verify the targeting of MNPs@Cur, the targeting effect was observed in activated HUVECs (Fig. 4c), where endothelial cells also play a major role in the inflammation initiation process. The targeting ability of MNPs@Cur could be significantly observed in both groups of inflammatory cells, indicating the chemotaxis of CCR-2 on the surface of macrophages to the site of inflammation, directing the binding to inflammatory cells. Meanwhile, it was also found that PNPs@Cur had relatively strong inflammatory targeting ability, and further compared with CNPs@Cur (Fig. 5b), the expected results were similar to the speculation also fitted indirectly with the guide theory of TCM. The intracellular fluorescence intensity of CNPs@Cur may be a result of the positive charge on the surface of their particles, allowing them to be readily taken up by various cells. These results suggested that PNPs also showed affinity for major 
components of inflammation-related cells in vitro, which may be attributed to the receptor/protein binding mechanism between the monosaccharide portion of PGP therein and inflammatory cells. Certainly, this speculation still needs further deeper excavation to provide stronger scientific support for the guide theory of Platycodon grandiflorum.

To further investigate the intracellular release behavior of the drug, NR fluorescent probes (which barely fluoresce in polar solutions but have intense red fluorescence in the lipid environment) were selected to observe the release state in inflammatory cells.MNPs@NR were co-incubated with activated macrophages. As shown in the figure, the red fluorescence of NR being excited in inflammatory cells is increasing in a time-dependent manner (Fig. 4d). Generally, the wrapping of macrophage membranes allowed MNPs to effectively avoid uptake by macrophages, thereby further enhanced targeting to inflammatory cells, and implied that MNPs@Cur could release drugs precisely at the site of inflammation, avoiding side effects at other sites. More astonishingly, PNPs also possess this kind of superiority compared to CNPs, only that their intrinsic mechanisms need to be studied more diligently. The ROSresponsive PNPs coated with macrophage membranes constructed in this study with dual targeting may provide a potential platform for improved therapeutic targeting of inflammation.

\section{In vitro assessment of safety and anti-inflammatory effects}

Then, the safety of PNPs as well as MNPs was verified by systematically assessing their biocompatibility in vitro. HUVECs and RAW264.7 cells were incubated with media containing different concentrations of PNPs and MNPs for 48 hours to assess their cytotoxicity. These results showed (Fig. 6a, b) favorable cytocompatibility in all experimental groups compared to the control group.

The other important indicator of biomaterials, blood compatibility, further confirmed the safety of the carrier material. Hemolysis is present in the relevant components of Platycodon grandiflorum. Therefore, PNPs as well as MNPs were evaluated in vitro to evaluate whether hemolysis could be avoided. Hemolysis of PNPs and MNPs was examined by direct contact method. As shown in Fig. 6c, PNPs and MNPs were not significantly different from the negative control, with hemolysis rates even lower than $0.5 \%$ (Fig. $6 \mathrm{~d}$ ). Considering the above, the results of cytotoxicity as well as hemocompatibility confirmed the superior biocompatibility of the carriers.

The cytokines and pro-inflammatory mediators released by macrophages play an important role in the pathogenesis of ALI[44]. Cur is a natural phenolic compound with various pharmacological effects such as anti-inflammatory, antioxidant, anti-proliferative and anti-angiogenic. The appearance of cytokine storm is an important reason for the sudden exacerbation of the disease in many COVID-19 patients. Therefore, blocking cytokine storms is one of the key strategies to save patients' lives. It has been reported that IL-6 and TNF-a are key factors in triggering inflammatory storms $[9,10]$. As shown in the Fig.6e, we examined the blocking ability of MNPs@Cur on cytokine storm at the cellular level and could observe that the expression levels of TNF- $a$ and IL- 6 were downregulated in all treatment groups, indicating the anti-inflammatory ability of Cur. Compared to the free drug, MNPs@Cur and PNPs@Cur 
downregulated levels were nearer to those of the control group (Fig. 6f), indicating a substantial intracellular release and thus efficacy of the drug consistent with the previous cellular targeting results. Collectively, these results demonstrated the superior biocompatibility of PNPs and MNPs and further showed the excellent targeting ability of the constructed nanosystems on inflammatory cells to calm the cytokine storm.

\section{Biodistribution in vivo}

Given that MNPs exhibit significant ability to target inflammatory cells in vitro, we next investigated whether the lungs of inflamed mice could be specifically targeted in vivo. ALI mice were constructed and free DiR, CNPs@DiR, PNPs@DiR and MNPs@DiR (same dose of DiR) were injected intravenously into the mice in random groups, with healthy mice as controls. After $3 \mathrm{~h}$ of administration, the lung tissues collected from each group were imaged by IVIS, and it was observed (Fig. 7a) that MNPs@DiR showed the strongest fluorescence intensity in the lungs of inflamed mice compared with the fluorescence in the lungs of healthy mice or ALI mice from other dosing treatment groups, indicating that MNPs@DiR specifically targets the inflamed lungs of mice. Notably, the fluorescence intensity of PNPs@DiR was secondonly to that of MNPs@DiR (Fig. 7c). Consistent with previous cellular targeting results, the stronger fluorescence intensity of PNPs@DiR compared with DiR and CNPs@DiR implies that PNPs have the potential function of guiding drugs into the damaged lungs more than CNPs. It was consistent with the theory that Platycodon grandiflorum guides drugs into the lung, implying that PGP may be one of the vital channel ushering components of Platycodon grandiflorum. Meanwhile, in the DiR-injected ALI mice, the fluorescent signal at $3 \mathrm{~h}$ was aggregated in the liver as well as the spleen. The weaker fluorescence intensity of DiR in PNPs@DiR, taken up by the liver and spleen, and the least fluorescence signal in MNPs@DiR-treated ALI mice was essentially present only in the liver, suggesting that after being coated by MM (Fig. 7b, d), MNPs are more likely to achieve immune escape and thus enhance the accumulation to inflamed lungs.

These results can be explained by the intrinsic affinity of macrophages for sites of inflammation. They can bind to activated/inflamed vessel walls through a range of receptor modes, including the chemokine receptor CCR2 and adhesion factors on the cell membrane surface. Furthermore, the temporary vasodilation and leakage of blood vessels induced by histamine at the time of injury allows injected nanoscale MNPs to passively target inflamed lung tissue through the vessels to the injured tissue. Regarding the pulmonary targeting ability of PNPs, we hypothesized that the monosaccharide component of PGP binds to receptors overexpressed on the surface of affected lung tissues and possesses the ability of PNPs to deliver drugs to the lung, and the specific targeting mechanism still needs further study. Conclusively, the ROS-responsive PNPs coated with macrophage membranes constructed in this study possessing dual targeting may provide a potential targeting delivery platform for improving the treatment of lung inflammation.

\section{Improved treatment of acute pneumonia with MNPs@Cur}


As previously mentioned, prevention or inhibition of cytokine storm may be one of the key strategies to save the lives of patients with severe pneumonia. Therefore, we investigated whether cytokine storms could be calmed by targeting MNPs@Cur to pneumonia sites. Figure 8a shows the experimental protocol. To verify the efficacy of model drug-targeted delivery, mice were treated with LPS ( $10 \mathrm{mg} / \mathrm{kg}$ ) to induce ALI. $5 \mathrm{~h}$ later, saline, free Cur (30 mg/kg),PNPs@Cur and MNPs@Cur (equivalent to $30 \mathrm{mg} / \mathrm{kg}$ of Cur) were injected intravenously and all mice were euthanized $19 \mathrm{~h}$ after injection for analysis. The serum levels of TNF- $a$ and IL- 6 in the different treatment groups were analyzed separately using ELISA kits. Although Cur has been shown to reduce cytokine storm in previous studies, in our experimental mouse model of ALI, Cur had limited therapeutic effect and treatment with free curcumin modestly reduced cytokine levels. Encouragingly, treatment with MNPs@Cur resulted in a significant decrease in these inflammatory factors compared to the free drug group, demonstrating that targeted delivery of MNPs@Cur could effectively inhibit cytokine storm (Fig. 8b, c), which again confirms the superior inflammatory lung targeting ability of this combined dual-targeting bionic carrier.

In addition, we further measured the wet/dry weight ratio to observe the pulmonary edema. Pathological examination revealed edema in the lungs of COVID-19 patients, which may be due to excessive mucus secretion and infiltration of immune cells in the lungs. In the ALI mouse, we found excessive pulmonary edema, alveolar inflammatory cell exudation/infiltration and alveolar damage in lung tissue sections. By administration of different formulations and free drugs, we found that treatment with MNPs@Cur significantly reduced inflammatory cell infiltration in mice compared to the free drug group (Fig. 8g, h). Meanwhile in the MNPs@Cur treatment group markedly improved the pulmonary edema status (Fig. 8d), and the wet/dry weight ratio of the lungs was more inclined to the normal group.

As a marker of neutrophil accumulation in inflammatory tissues, myeloperoxidase (MPO) levels can be assessed for the degree of migration and infiltration of neutrophils in inflammatory cells. As shown in the Fig. 8e, the MNPs@Cur treatment group remarkably repressed the migration and infiltration of inflammatory cells, which corresponded to the improvement of pulmonary edema. Given that oxidative stress is also part of the pathogenesis of ALI, malondialdehyde (MDA), a lipid peroxidation product, can reflect the level of ROS production from lipid oxidation. It was found that MDA was significantly elevated in the saline group Fig. 8f, indicating a redox imbalance and causing severe oxidative damage in AlL mice, and yetMNPs@Cur significantly inhibited the production of MDA compared with other groups, thus improving the microenvironment of oxidative stress in vivo. Above results showed that ROS were effectively eliminated in vivo by MPO and MDA, which are important defenses to mediate ROS in impaired lungs. The results of MPO and MDA, which are important defenses to neutralize impaired pulmonary ROS, indicated that the ROS were effectively cleared in vivo. Generally, the bionic drug delivery systems MNPs@Cur based on PGP can alleviate lung inflammation effectively, which means that MNPs can improve the pulmonary delivery of drugs and hence facilitate ALI treatment. Meanwhile, the results of H\&E staining also showed no significant damage to major organs and immunotoxicity after treatment, which further validated the beneficial biocompatibility of our constructed drug delivery platform in vivo (Fig. 9). 
Pneumonia is a serious respiratory disease that leads to high morbidity and mortality. Currently, few targeted therapeutic strategies are available, as specific delivery of drugs to the inflamed lungs remains challenging[45]. Although significant efforts have been made to develop effective pulmonary drug delivery systems, there is still a lack of nanodrug delivery systems that target the pathogenesis of lung inflammation. Combined with the presence of overexpressed ROS at the lesion site during pneumonia, this would allow pro-inflammatory factors to recruit macrophages to the lesion site as well as the guide theory of TCM. Therefore, we developed a ROS-responsive nanoparticle based on PGPs and coated MM on the surface of the nanoparticle to construct a bionic drug delivery system for lung diseases.

Experiments were conducted sequentially at the cellular as well as animal levels to verify the excellent lung targeting ability and precise drug release. It provides inspiration for exploring other channel ushering components to construct natural drug targeting systems, and provides a strong experimental support for the modernization of Chinese medicine guide theory.

\section{Conclusion}

Collectively, inspired by the inherent affinity of macrophages for the site of inflammation and the guide theory of Platycodon grandiflorum. We have developed a PGP-based bionic drug delivery platform for the treatment of pneumonia. We found that MNPs can accumulate at sites of inflammation associated with pneumonia and facilitate delivery of anti-inflammatory drugs via intravenous administration. It is emphasized by our results that targeted drug delivery for pneumonia significantly reduces cytokine storm syndrome compared to free drug therapy. The prepared dual-targeted drug delivery platform perfectly combines modern bionic technology and TCM concepts and may provide a promising approach for the treatment of patients with COVID-19. Since the excellent biocompatibility and accessibility to preparation, it would provide a strong scientific basis for related TCM classical prescriptions and facilitates the search for natural targeted drug delivery systems, showing potential for further clinical translation.

\section{Declarations}

\section{Acknowledgements}

All authors acknowledge financial support from Taishan Scholar Foundation of Shandong Province (No. qnts20161035), Natural Science Foundation of Shandong Province (No. ZR2019ZD24, ZR2019YQ30), Graduate Innovation Foundation of Yantai University, GIFYTU. We would also like to thank Editage (www.editage.cn) for English language editing.

\section{Authors' contributions}

$Y L, C G$ and DC conceived of this study and designed it. YL and CG contributed to data collection and article writing. QC, YS and HG performed the preparation of the references in the manuscript. All authors read and approved the final manuscript.

\section{Funding}


This funding was supported by Taishan Scholar Foundation of Shandong Province (No. qnts20161035); Natural Science Foundation of Shandong Province (No. ZR2019ZD24, ZR2019YQ30).

\section{Availability of data and materials}

All data generated or analyzed in this study are included in this article.

\section{Ethics approval and consent to participate}

All procedures involving laboratory animals are performed in accordance with the ethics committee guidelines at the Yantai University.

\section{Consent for publication}

Not applicable.

\section{Competing interests}

The authors declare no conflict of interest in this article.

\section{References}

1. Butt Y, Kurdowska A, Allen TCJAoP, Medicine L. Acute Lung Injury: A Clinical and Molecular Review. 2016, 140:345-350.

2. Dinarello CAJC. Anti-inflammatory Agents: Present and Future. 2010, 140:935-950.

3. Mcvey MJ, Steinberg BE, Goldenberg NMJALC, Physiology M. Inflammasome Activation in Acute Lung Injury. 2020, 320.

4. Medzhitov RJN. Origin and physiological roles of inflammation. 2008, 454:428-435.

5. Fajgenbaum DC, June CHJTNEjom. Cytokine Storm. Reply. 384:e59.

6. Nile SH, Nile A, Qiu J, Li L, Kai GJC, Reviews GF. COVID-19: Pathogenesis, cytokine storm and therapeutic potential of interferons. 2020, 53.

7. Ragab D, Eldin HS, Taeimah M, Khattab R, Salem RJFil. The COVID-19 Cytokine Storm; What We Know So Far. 2020, 11:1446.

8. Tang L, Yin Z, Hu Y, Mei HJFil: Controlling Cytokine Storm Is Vital in COVID-19. 2020, 11.

9. Copaescu FA, Smibert FO, Ag B, Elizabeth J. Phillips MD F, FRACP b c, Jason A. Trubiano MBBS B, PhD, FRACP a d e $\mathrm{f} \% \mathrm{~J}$ Journal of Allergy, Immunology C: The role of IL- 6 and other mediators in the cytokine storm associated with SARS-CoV-2 infection. 2020, 146:535-536.

10. Karki R, Sharma BR, Tuladhar S, Williams EP, Zalduondo L, Samir P, Zheng M, Sundaram B, Banoth B, Malireddi RJC: Synergism of TNF-a and IFN- $y$ Triggers Inflammatory Cell Death, Tissue Damage, and Mortality in SARS-CoV-2 Infection and Cytokine Shock Syndromes. 2020. 
11. Hu Y, Wang T, Hu Z, Wang X, Peng PJB, Pharmacotherapy. Clinical efficacy of glucocorticoid on the treatment of patients with COVID-19 pneumonia: A single-center experience. 2020, 130:110529.

12. Siemieniuk RA, Bartoszko JJ, Long G, Zeraatkar D, Brignardello-Petersen RJB: Drug treatments for covid-19: living systematic review and network meta-analysis. 2020, 370:m2980.

13. Steroid therapy and the risk of osteonecrosis in SARS patients: a dose-response meta-analysis \%J Osteoporosis International. 2016, 28:1027-1034.

14. Caplan A, Fett N, Rosenbach M, Werth VP, Micheletti RGJJotAAoD: Prevention and management of glucocorticoid-induced side effects: A comprehensive review: Infectious complications and vaccination recommendations. 2017.

15. Oray M, Abusamra K, Ebrahimiadib N, Meese H. Foster CSJEOoDS: Long-term side effects of glucocorticoids. 2016, 15:457-465.

16. Evolving functions of endothelial cells in inflammation. \%J Nature Reviews Immunology. 2007, 7:803-815.

17. Mata R, Yao Y, Cao W, Ding J, Gao CJR. The Dynamic Inflammatory Tissue Microenvironment: Signality and Disease Therapy by Biomaterials. 2021, 2021:1-31.

18. Sfanos KS, Yegnasubramanian S, Nelson WG, Marzo ADJNRU. The inflammatory microenvironment and microbiome in prostate cancer development. 2018.

19. Chen J, Wang YK, Gao Y, Hu LS, Cao YBJB. Pharmacotherapy: Protection against COVID-19 injury by Qingfei Paidu decoction via anti-viral, anti-inflammatory activity and metabolic programming. 2020, 129:110281.

20. Xin S, Cheng X, Zhu B, Liao X, Yu RJB. Pharmacotherapy: Clinical Retrospective Study on the Efficacy of Qingfei Paidu Decoction Combined with Western Medicine for COVID-19 Treatment. 2020, 129:110500.

21. Li S, Liu C, Guo F, Taleb SJ, Tong M, Shang DJTAJoCM: Traditional Chinese Medicine as Potential Therapy for COVID-19. 2020.

22. Ren JL, Zhang AH, Wang XJJPR. Traditional Chinese medicine for COVID-19 treatment. 2020, 155:104743.

23. Zhao Z, Li Y, Zhou L, Zhou X, Sun JJP. Prevention and treatment of COVID-19 using Traditional Chinese Medicine: A review. 2020:153308.

24. Rattis B, Ramos SG, Celes MJFiP. Curcumin as a Potential Treatment for COVID-19. 2021, 12:675287.

25. Guo C, Hou X, Liu Y, Zhang Y, Chen DJP. Novel Chinese Angelica Polysaccharide Biomimetic Nanomedicine to Curcumin Delivery for Hepatocellular Carcinoma Treatment and Immunomodulatory Effect. 2021, 80:153356.

26. Sheng Y, Liu G, Wang M, Lv Z, Du PJIJoBM. A selenium polysaccharide from Platycodon grandiflorum rescues PC12 cell death caused by H2O2 via inhibiting oxidative stress. 2017, 104:393-399. 
27. Zhao X, Wang Y, Peng Y, Cheng G, Wang C, Geng N, Wang X, Liu JJM. Effects of Polysaccharides from Platycodon grandiflorum on Immunity-Enhancing Activity In Vitro. 2017, 22.

28. Ci T, Li H, Chen G, Wang Z, Gu ZJSA. Cryo-shocked cancer cells for targeted drug delivery and vaccination. 2020, 6:eabc3013.

29. Ma Q, Fan Q, Xu J, Bai J, Wang CJM. Calming Cytokine Storm in Pneumonia by Targeted Delivery of TPCA-1 Using Platelet-Derived Extracellular Vesicles. 2020, 3.

30. Tang S, Zhang F, Gong H, Wei F, Wang JJSR. Enzyme-powered Janus platelet cell robots for active and targeted drug delivery. 2020, 5:eaba6137.

31. Xue J, Zhao Z, Lei Z, Xue L, Zhang CJNN. Neutrophil-mediated anticancer drug delivery for suppression of postoperative malignant glioma recurrence. 2017, 12:692.

32. Zhang W, Wang M, Tang W, Wen R, Zhou S, Lee C, Wang H, Jiang W, Delahunty IM, Zhen ZJAM: NanoparticleLaden Macrophages for Tumor-Tropic Drug Delivery. 2018, 30:1805557.18055511805557.1805511.

33. Kuang S, He F, Liu G, Sun X, Zhang MJB. CCR2-engineered mesenchymal stromal cells accelerate diabetic wound healing by restoring immunological homeostasis. 2021:120963.

34. Nywening TM, Belt BA, Cullinan DR, Panni RZ, Han BJ, Sanford DE, Jacobs RC, Ye J, Patel AA, Gillanders WEJG. Targeting both tumour-associated CXCR2 + neutrophils and CCR2 + macrophages disrupts myeloid recruitment and improves chemotherapeutic responses in pancreatic ductal adenocarcinoma. 2018:gutjnl-2017-313738.

35. Gao C, Huang Q, Liu C, Kwong C, Wang RJNC. Treatment of atherosclerosis by macrophagebiomimetic nanoparticles via targeted pharmacotherapy and sequestration of proinflammatory cytokines. 2020, 11.

36. Wang Y, Zhang K, Li T, Maruf A, Qin X, Luo L, Zhong Y, Qiu J, McGinty S, Pontrelli G, et al. Macrophage membrane functionalized biomimetic nanoparticles for targeted anti-atherosclerosis applications. Theranostics. 2021;11:164-80.

37. Wei X, Ran D, Campeau A, Xiao C, Zhang LJNL. Multiantigenic Nanotoxoids for Antivirulence Vaccination against Antibiotic-Resistant Gram-Negative Bacteria. 2019, 19.

38. Guo J, Li D, Tao H, Li G, Liu R, Dou Y, Jin T, Li L, Huang J, Hu H, Zhang J. Cyclodextrin-Derived Intrinsically Bioactive Nanoparticles for Treatment of Acute and Chronic Inflammatory Diseases. Adv Mater. 2019;31:e1904607.

39. Cao H, Dan Z, He X, Zhang Z, Yu H, Yin Q, Li YJAN. Liposomes Coated with Isolated Macrophage Membrane Can Target Lung Metastasis of Breast Cancer. 2016, 10:7738.

40. Tang M, Ping H, Qiang Z, Nanobiotechnology NTJJo. Polymeric micelles with dual thermal and reactive oxygen species (ROS)-responsiveness for inflammatory cancer cell delivery. 2017, 15.

41. Diwanji N, Bergmann AJNC. Basement membrane damage by ROS- and JNK-mediated Mmp2 activation drives macrophage recruitment to overgrown tissue. 2020, 11. 
42. Nie Y, Wang Z, Chai G, Xiong Y, Zhao PJM. Dehydrocostus Lactone Suppresses LPS-induced Acute Lung Injury and Macrophage Activation through NF-KB Signaling Pathway Mediated by p38 MAPK and Akt. 2019, 24:1510.

43. Orecchioni M, Ghosheh Y, Pramod AB, Ley KJo. Macrophage Polarization: Different Gene Signatures in M1(LPS+) vs. Classically and M2(LPS-) vs. Alternatively Activated Macrophages. 2019, 10.

44. Debra L, Laskin, Rama, Malaviya, Toxicology DJJTsaojotSo: Role of Macrophages in Acute Lung Injury and Chronic Fibrosis Induced by Pulmonary Toxicants. 2018.

45. Fan E, Brodie D, Slutsky ASJJ. Acute Respiratory Distress Syndrome: Advances in Diagnosis and Treatment. 2018, 319:698-710.

\section{Figures}

a
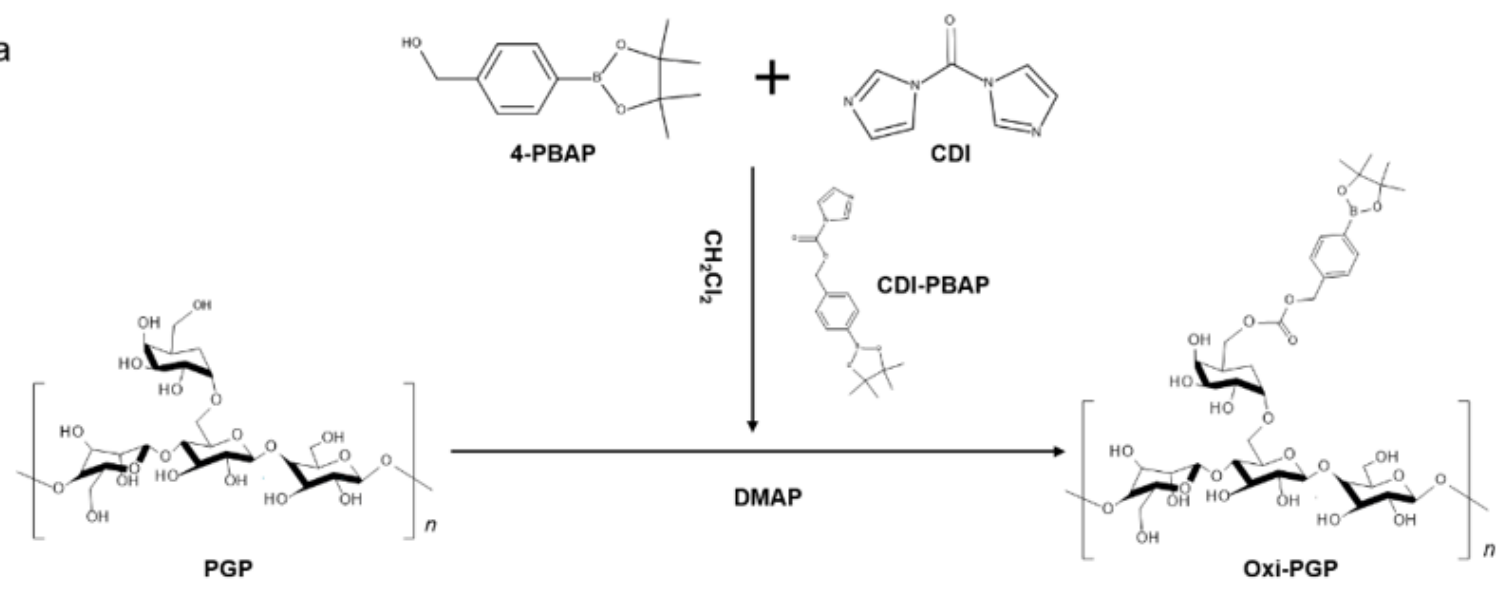

b
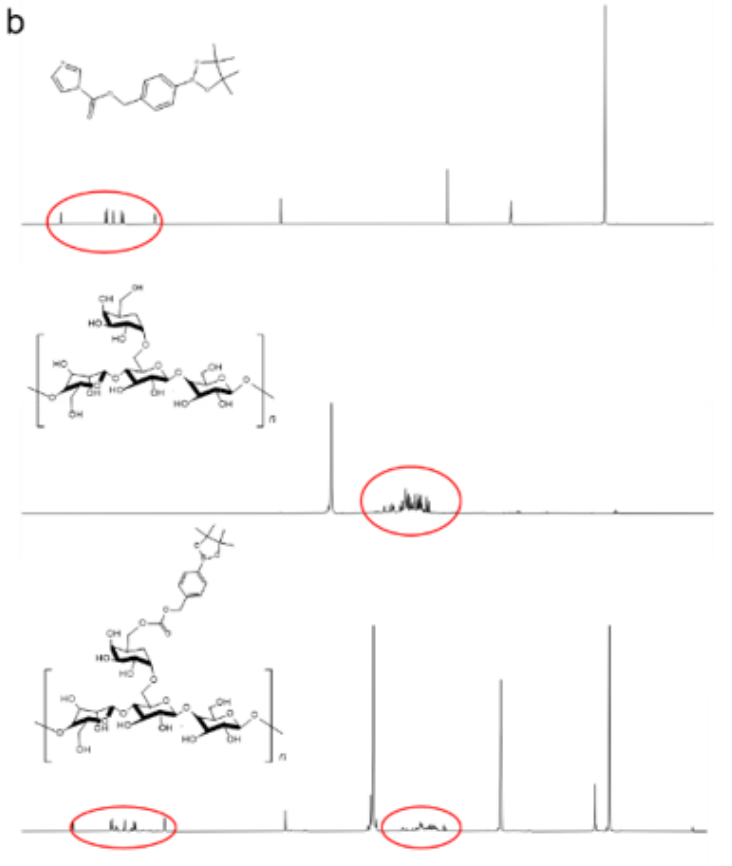

C

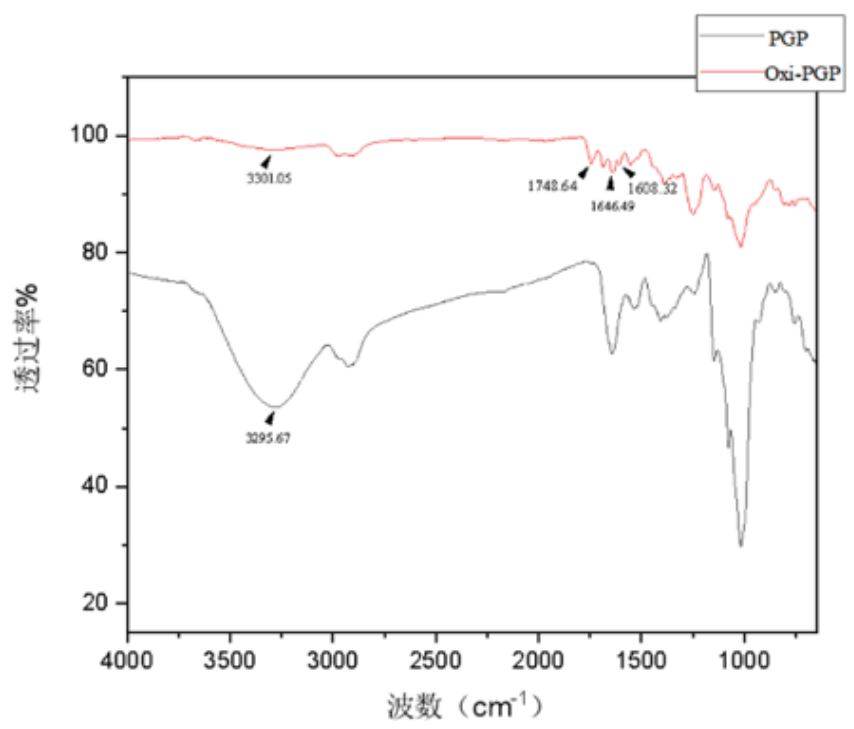


Figure 1

Synthesis and characterization of Oxi-PGP. a The synthesis route of Oxi-PGP. b 1H NMR spectrum of CDIPBAP, PGP and Oxi-PGP. c FT-IR spectrum of PGP and Oxi-PGP

a

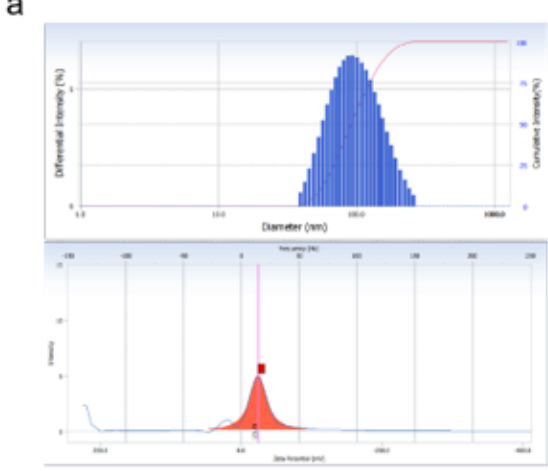

d

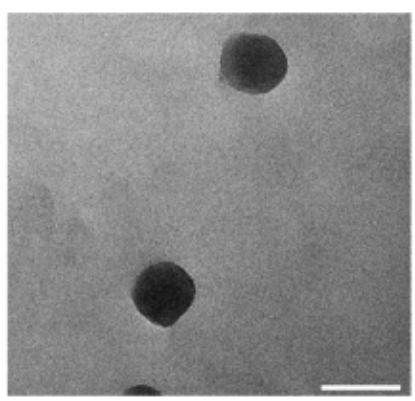

g

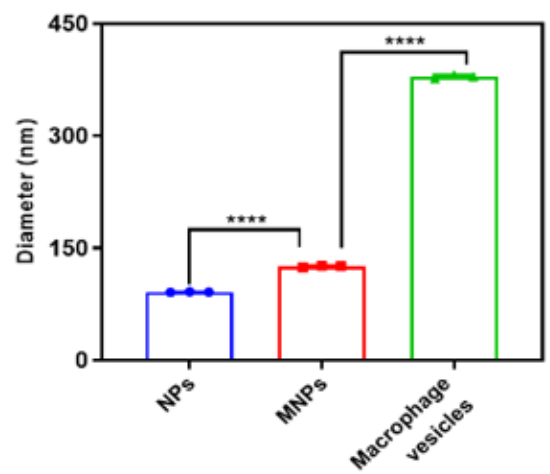

b

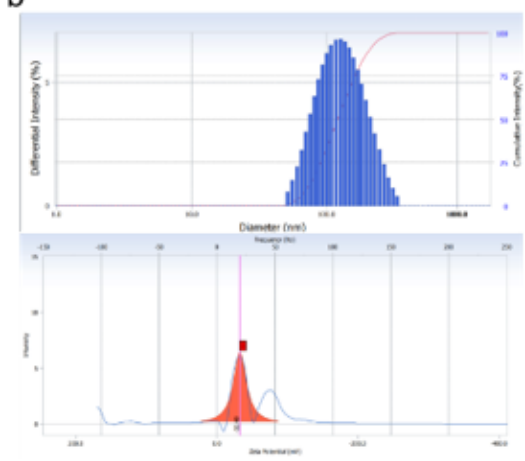

e

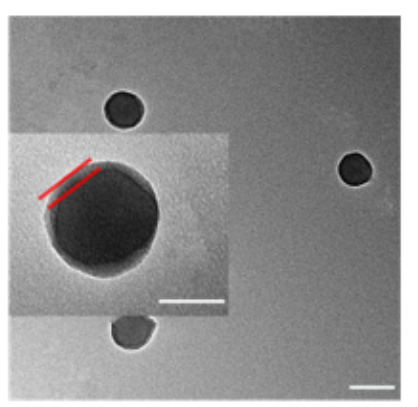

$\mathrm{h}$

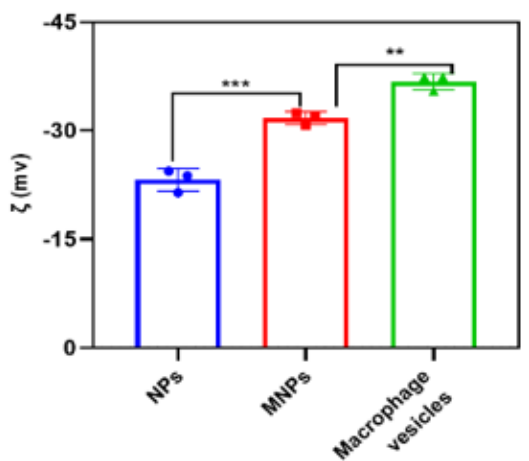

C

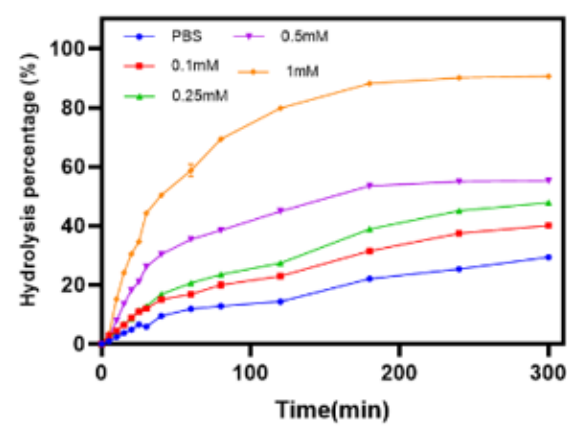

f

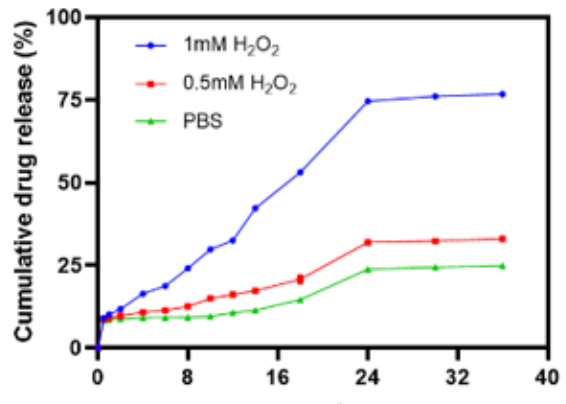

Time ( $h$ )

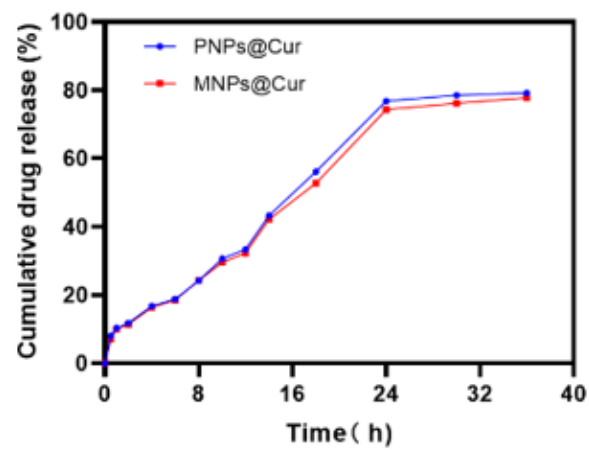

Figure 2

Preparation and characterization of ROS responsive PNPs and MNPs. $a, b$ The particle size and potential of the PNPs and MNPs respective DLS. c Hydrolysis rates of PNPs in PBS with different concentrations of $\mathrm{H} 2 \mathrm{O} 2(0,0.1 \mathrm{mM}, 0.25 \mathrm{mM}, 0.50 \mathrm{mM}$ and $1.00 \mathrm{mM})$. d TEM images of representative PNPs. Scale bar. $100 \mathrm{~nm}$. e TEM images of representative MNPs. Scale bar. $100 \mathrm{~nm}$. Inset: the amplified TEM image of a single MNP. Scale bar: $100 \mathrm{~nm}$. $\mathrm{f}$ In vitro release of Cur from PNPs in PBS at different concentrations of $\mathrm{H} 2 \mathrm{O} 2(0,0.5 \mathrm{mM}$ and $1 \mathrm{Mm}) . \mathrm{g}$, h Particle sizes and Zeta potentials of PNPs and MNPs analyzed by DLS. i In vitro drug release profiles of PNPs@Cur and MNPs@Cur. Statistical significance was calculated by one-way ANOVA $(n=3)$. P-value: ${ }^{*} P<0.05,{ }^{\star} P P<0.01$ and ${ }^{\star * \star} P<0.001$. Data are means $\pm S D$ 
a

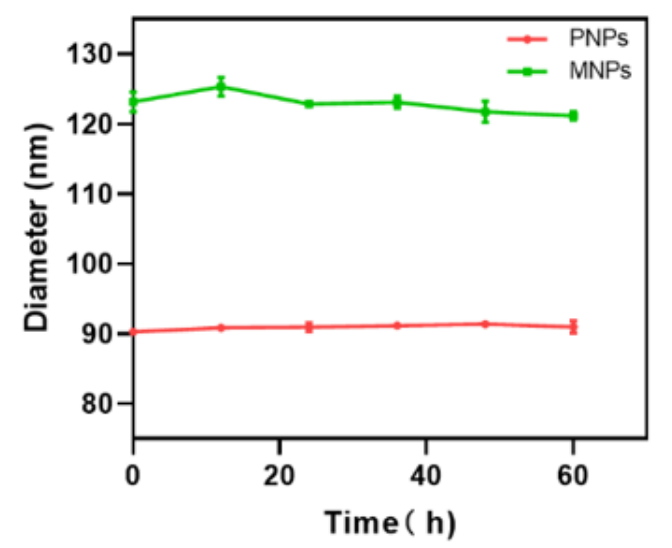

C

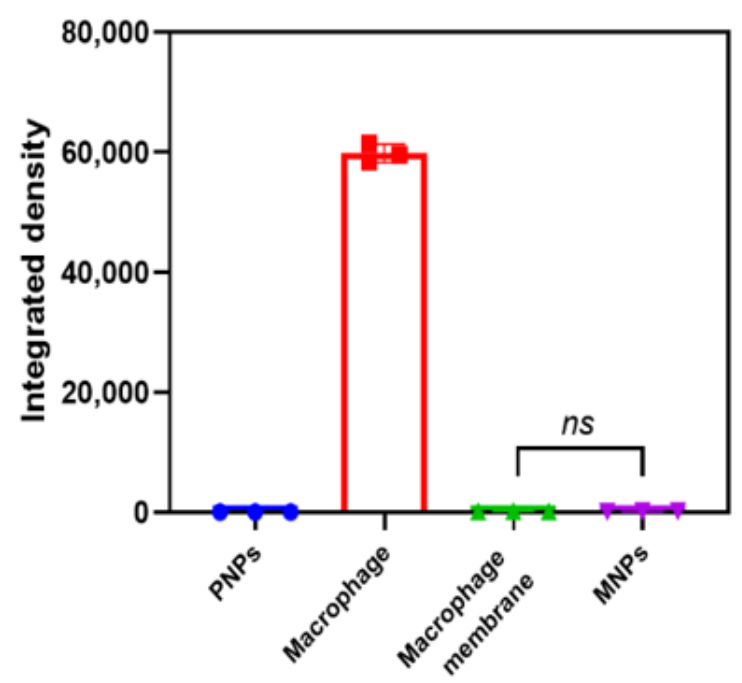

$\mathrm{b}$

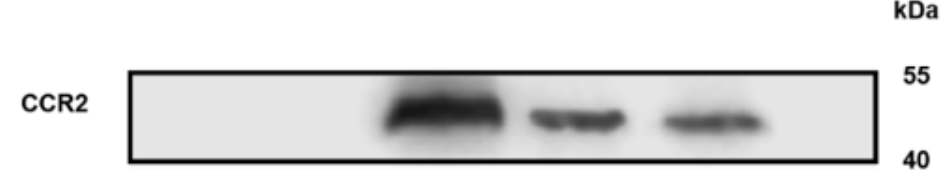

$\beta$-Actin

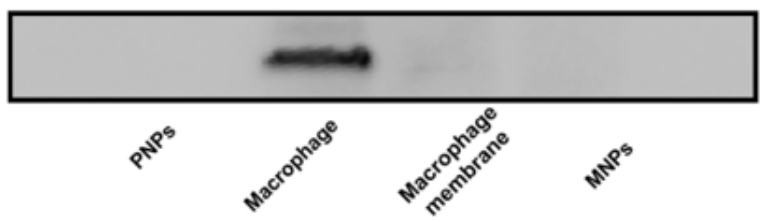

43

d

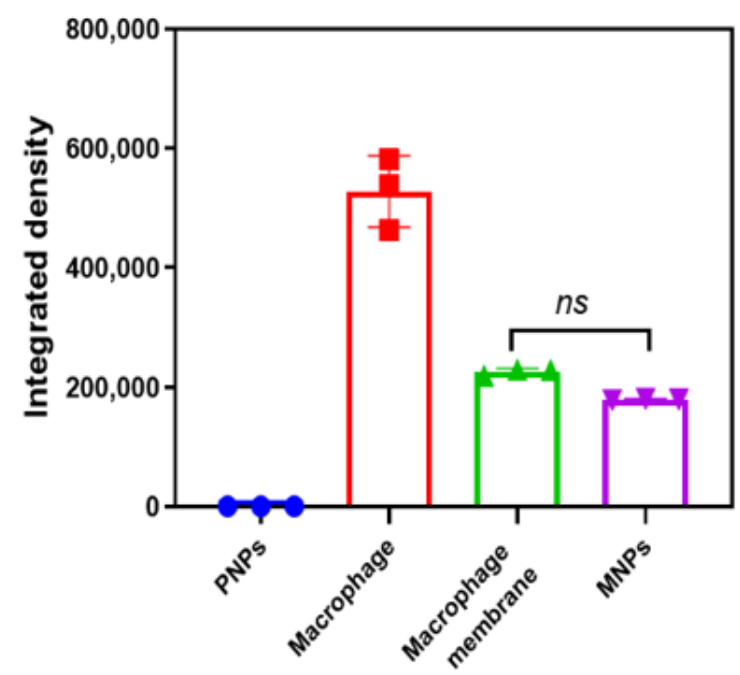

\section{Figure 3}

Stability and protein analysis. a. The particle stability of PNPs and MNPs incubated in the buffers for 60 $\mathrm{h}$ at $4^{\circ} \mathrm{C}$. b-d The protein bands characteristic of PNPs, macrophages, MM derived vesicles and MNPs were verified by Western blotting. Statistical significance was calculated by one-way ANOVA $(n=3)$. Pvalue: ${ }^{*} \mathrm{P}<0.05, * \star \mathrm{P}<0.01$ and $* \star * \mathrm{P}<0.001$. Data are means $\pm \mathrm{SD}$ 
a

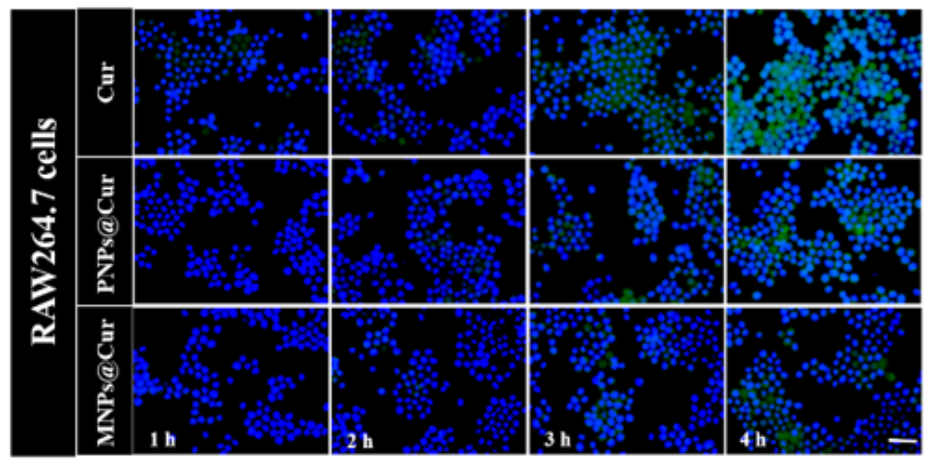

b
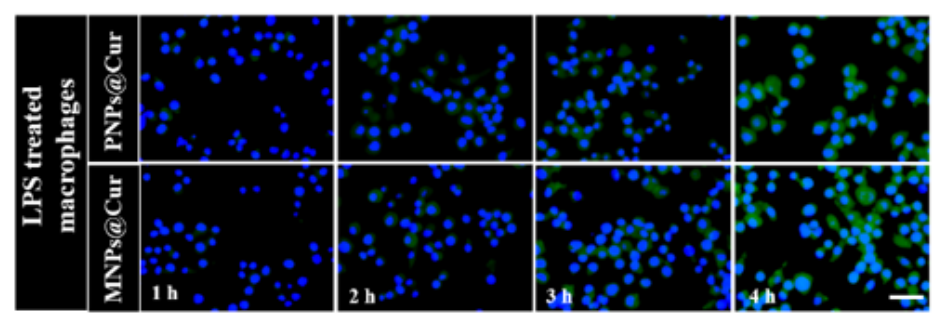

c

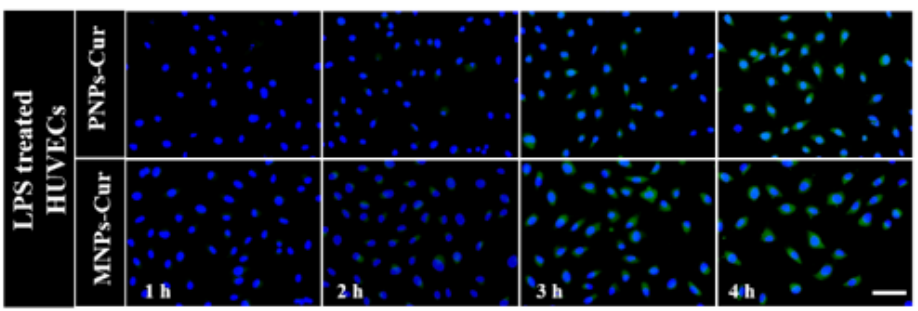

d

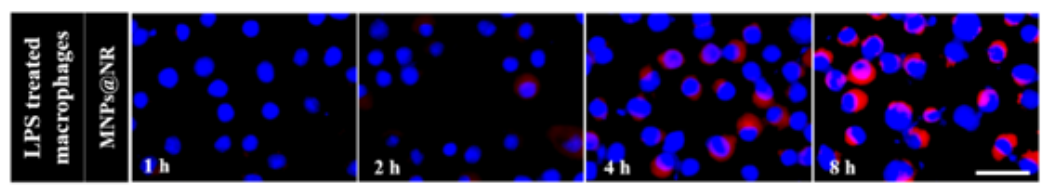

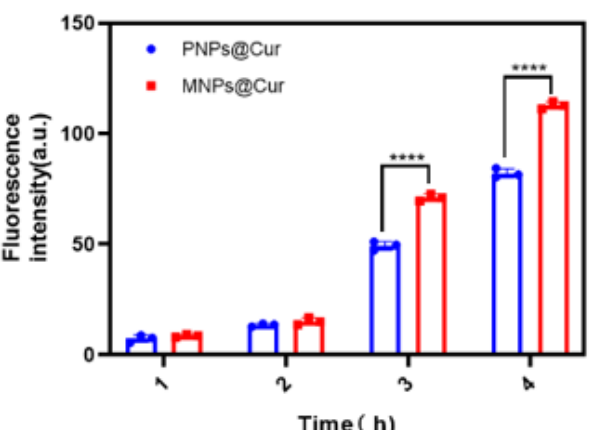
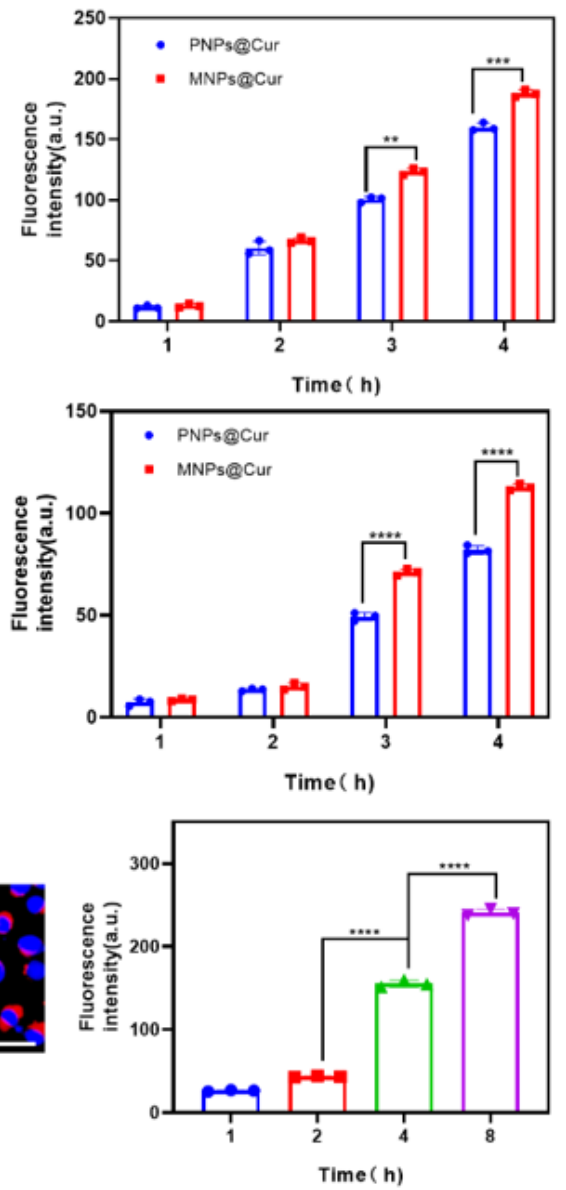

\section{Figure 4}

Capacity to target at the cellular level in vitro. a Ability to escape from RAW264.7 cells (including quantitative analysis) of free Cur,PNPs@Cur and MNPs@Cur. Scale bar: 50 mm. b, c Cellular uptake (including quantitative analysis) of PNPs@Cur and MNPs@Cur by RAW264.7 cells and HUVECs treated with LPS respectively. Scale bar: $50 \mu \mathrm{m}$. d Validated intracellular release of MNPs@NR in LPS-treated macrophages (including quantitative analysis). Scale bar: $50 \mu \mathrm{m}$. Statistical significance was calculated by one-way ANOVA and two-way ANOVA $(n=3-5)$. P-value: ${ }^{\star} P<0.05,{ }^{\star} * P<0.01$ and $* \star \star P<0.001$. Data are means \pm SD 

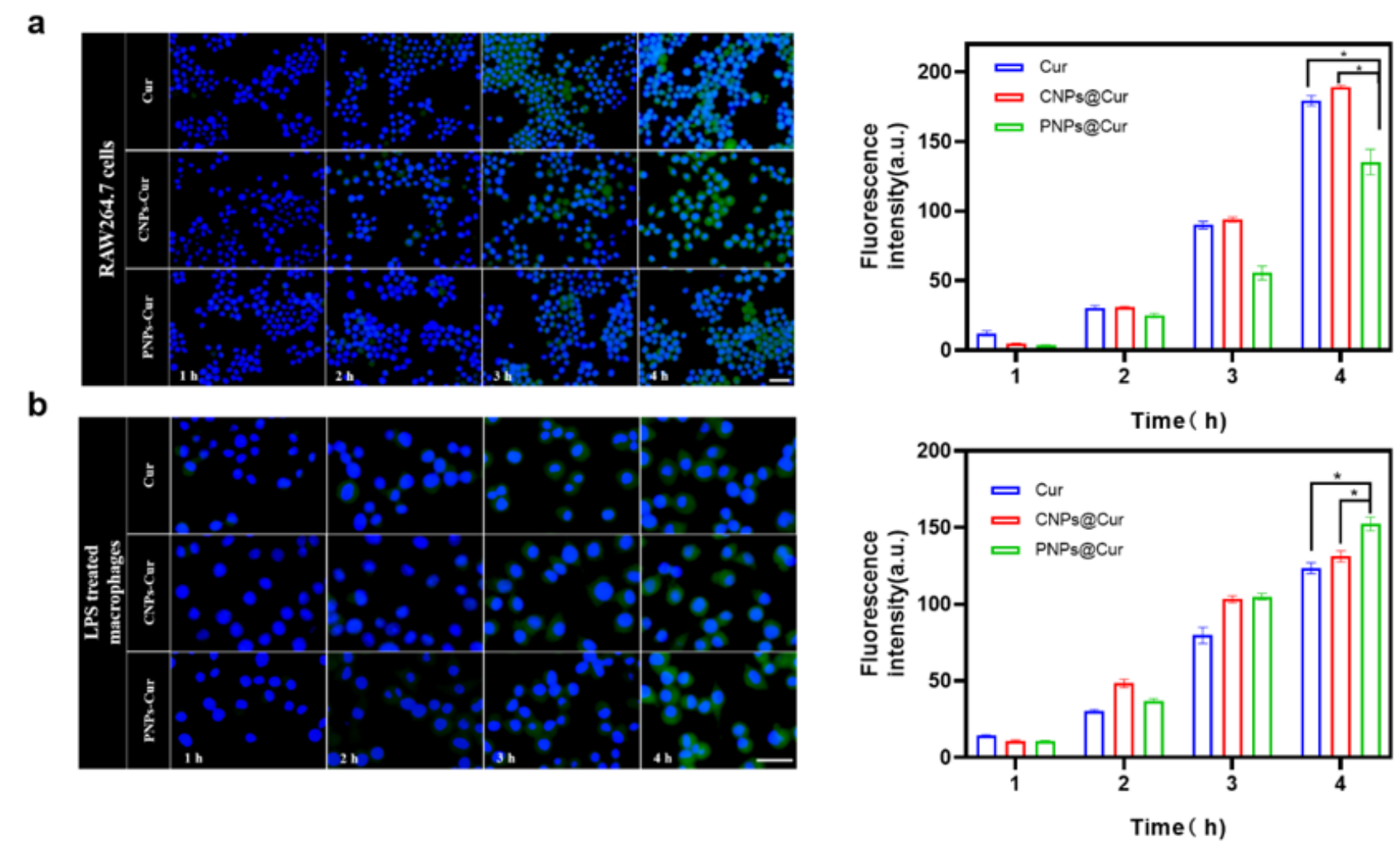

\section{Figure 5}

In vitro targeting of PNPs a Ability to escape from RAW264.7 cells (including quantitative analysis) of free Cur, CNPs@Cur and PNPs@Cur. Scale bar: 50 m. b Cellular uptake (including quantitative analysis) of

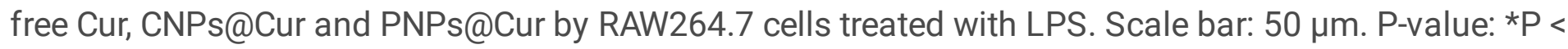
0.05. Data are means \pm SD 

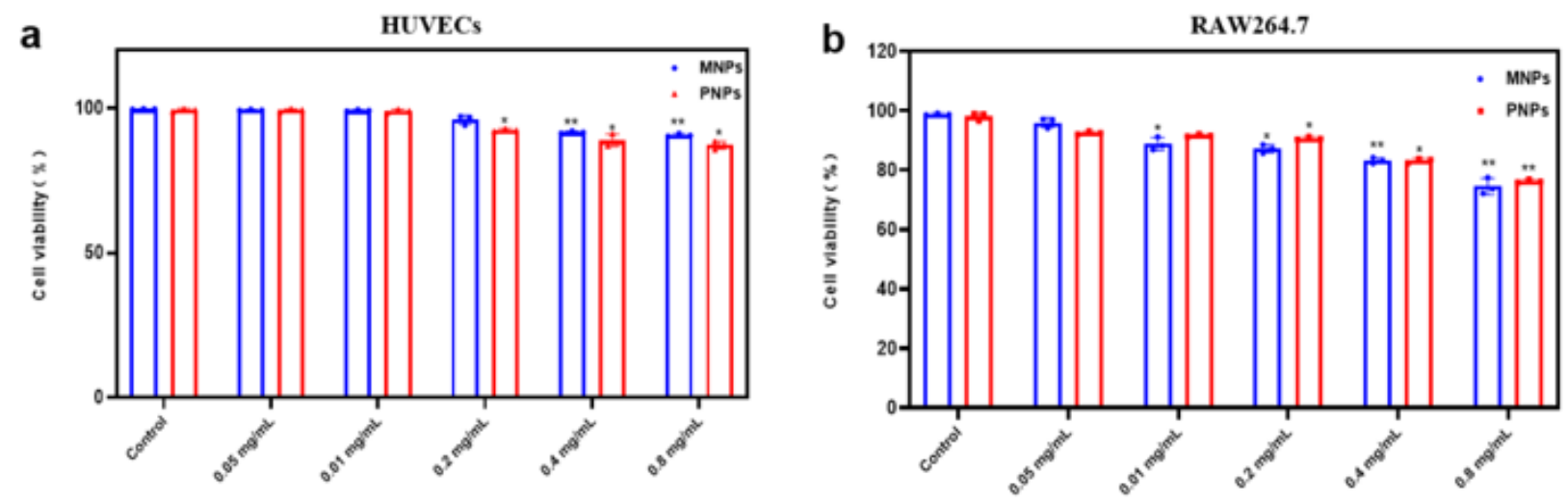

C

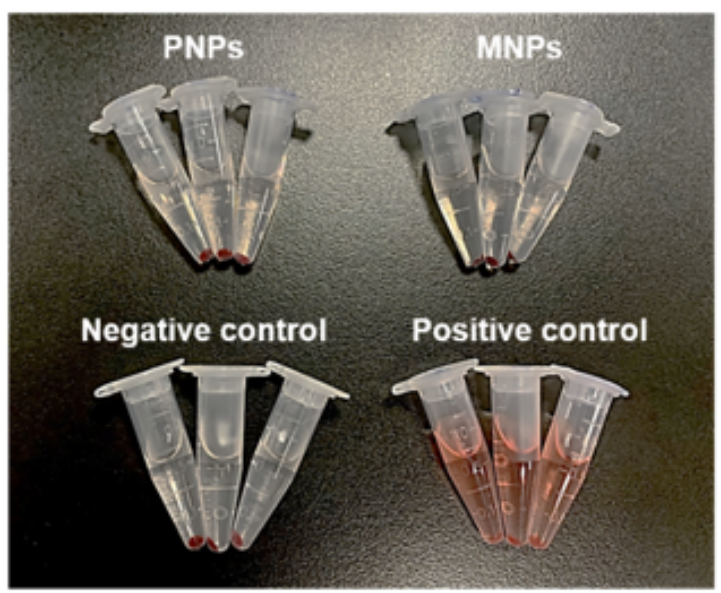

d

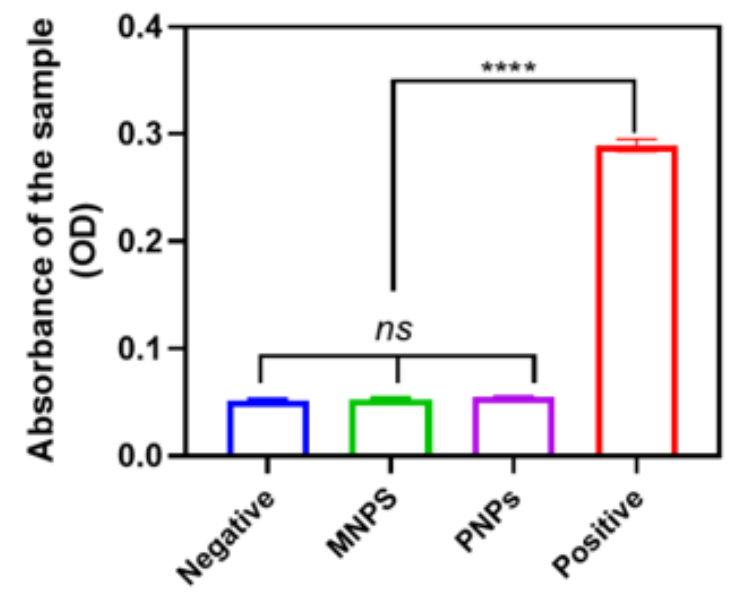

e
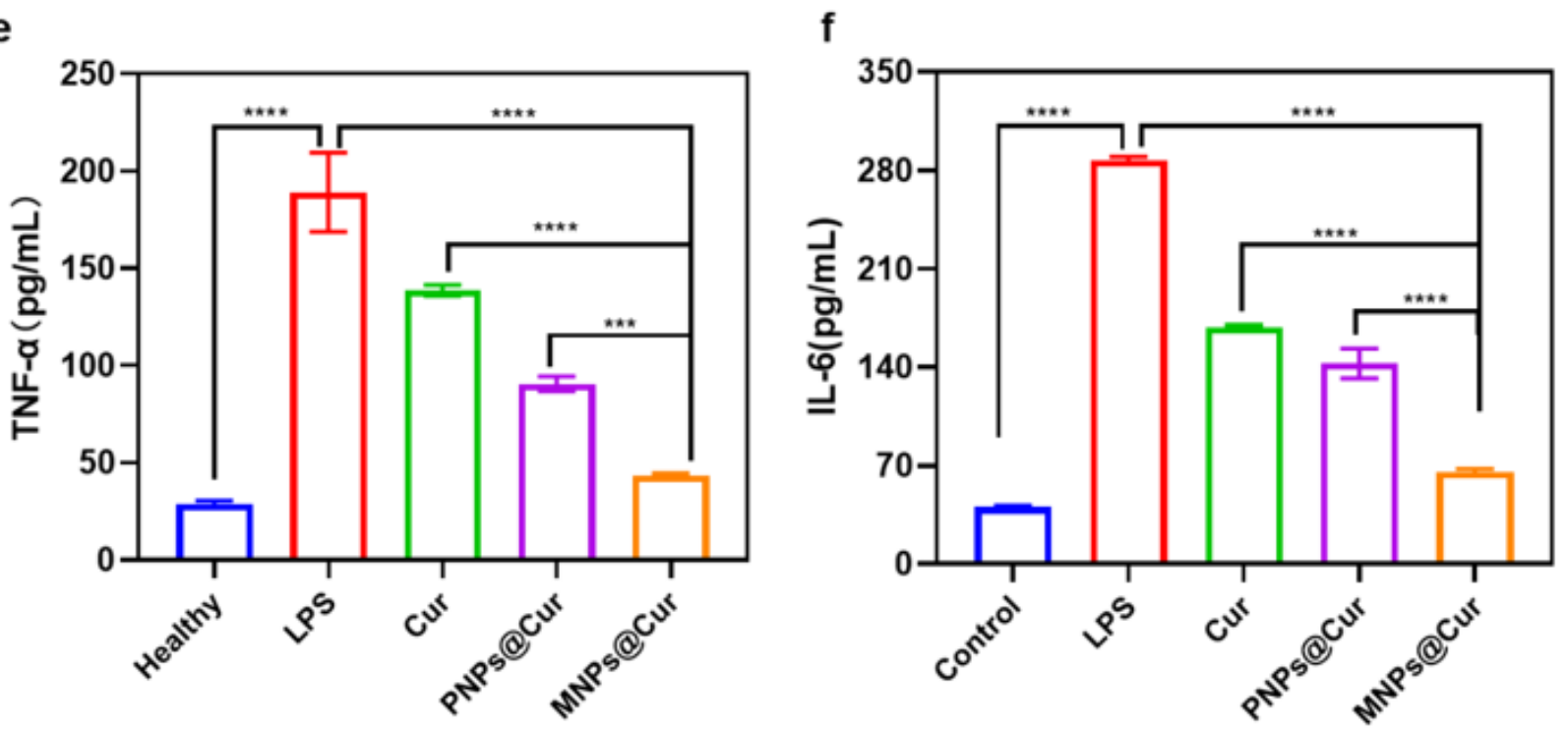

Figure 6

In vitro safety compatibility and suppression cytokine studies. a, b Cell viability of HUVECs andRAW264.7 cells after incubation with various doses of PNPs and MNPs for $48 \mathrm{~h}$. c Images of the hemolysis test with PNPs and MNPs. (D) The absorbance of PNPs and MNPs measured at $570 \mathrm{~nm}$. e, f Generation of TNF-a and IL-6 from activated macrophages supernatant after incubated with combinations of LPS (100 
ng/mL), free Cur, PNPs@Cur and MNPs@Cur. Statistical significance was calculated by two-way ANOVA and one-way ANOVA $(n=3)$. P-value: ${ }^{*} P<0.05,{ }^{*} \mathrm{P}<0.01$ and $* \star * P<0.001$. Data are means $\pm S D$

a

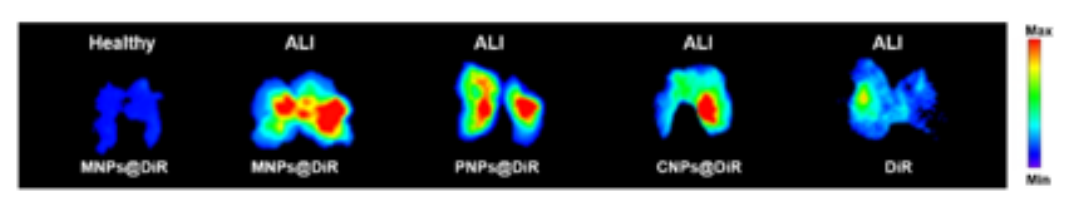

b

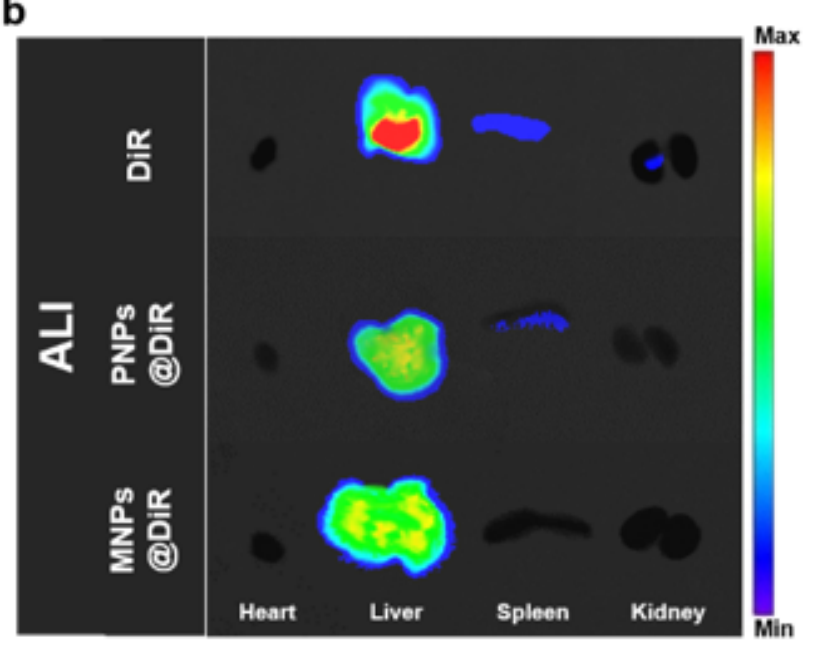

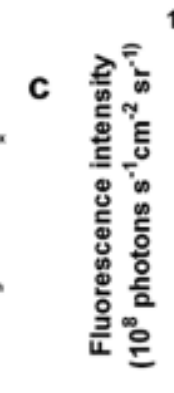
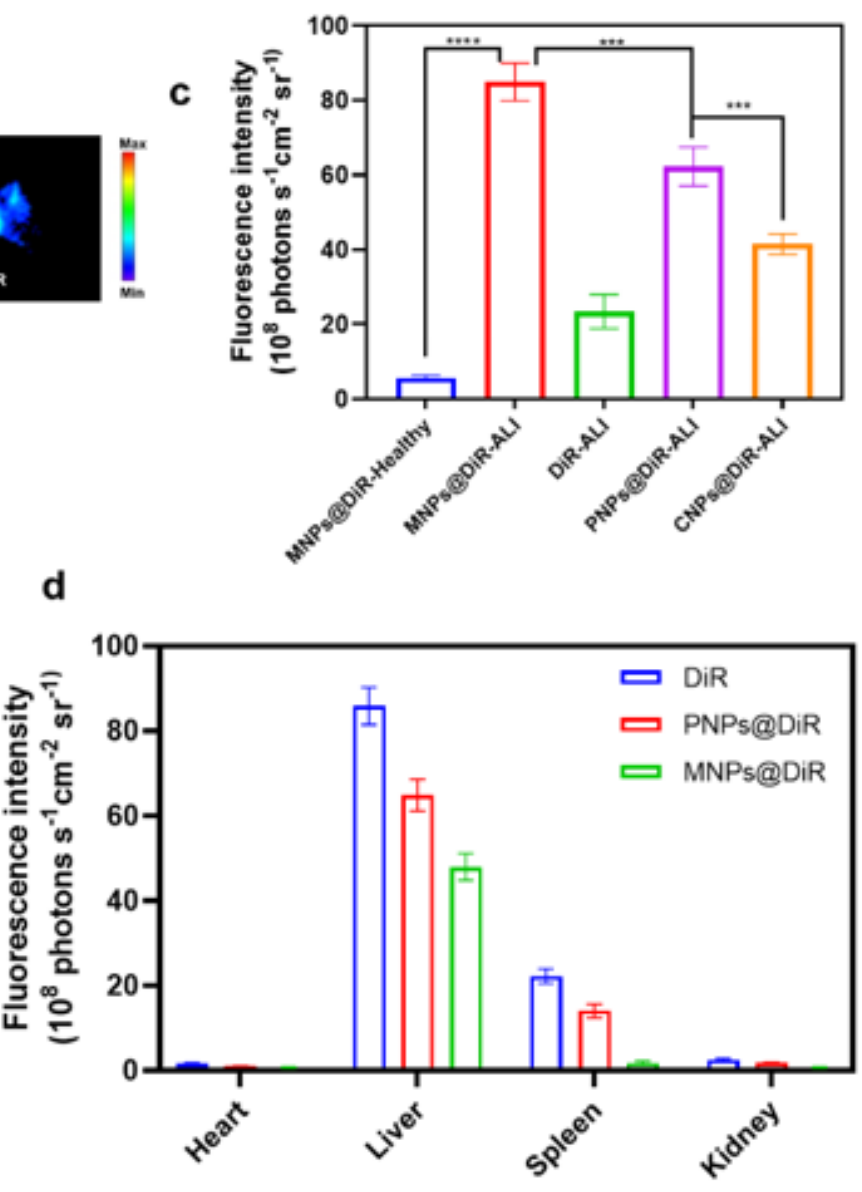

\section{Figure 7}

Targeting of impaired lungs in mice. a, b The biodistribution fluorescence of MNPs@DiR, MNPs@DiR and MNPs@DiR were measured using IVIS. c, d Quantitative analysis in lungs and other organs confirmed by IVIS. Statistical significance was calculated by two-way ANOVA and one-way ANOVA ( $n=3-5)$. P-value: *P $<0.05, * * P<0.01$ and $* \star * P<0.001$. Data are means \pm SD 

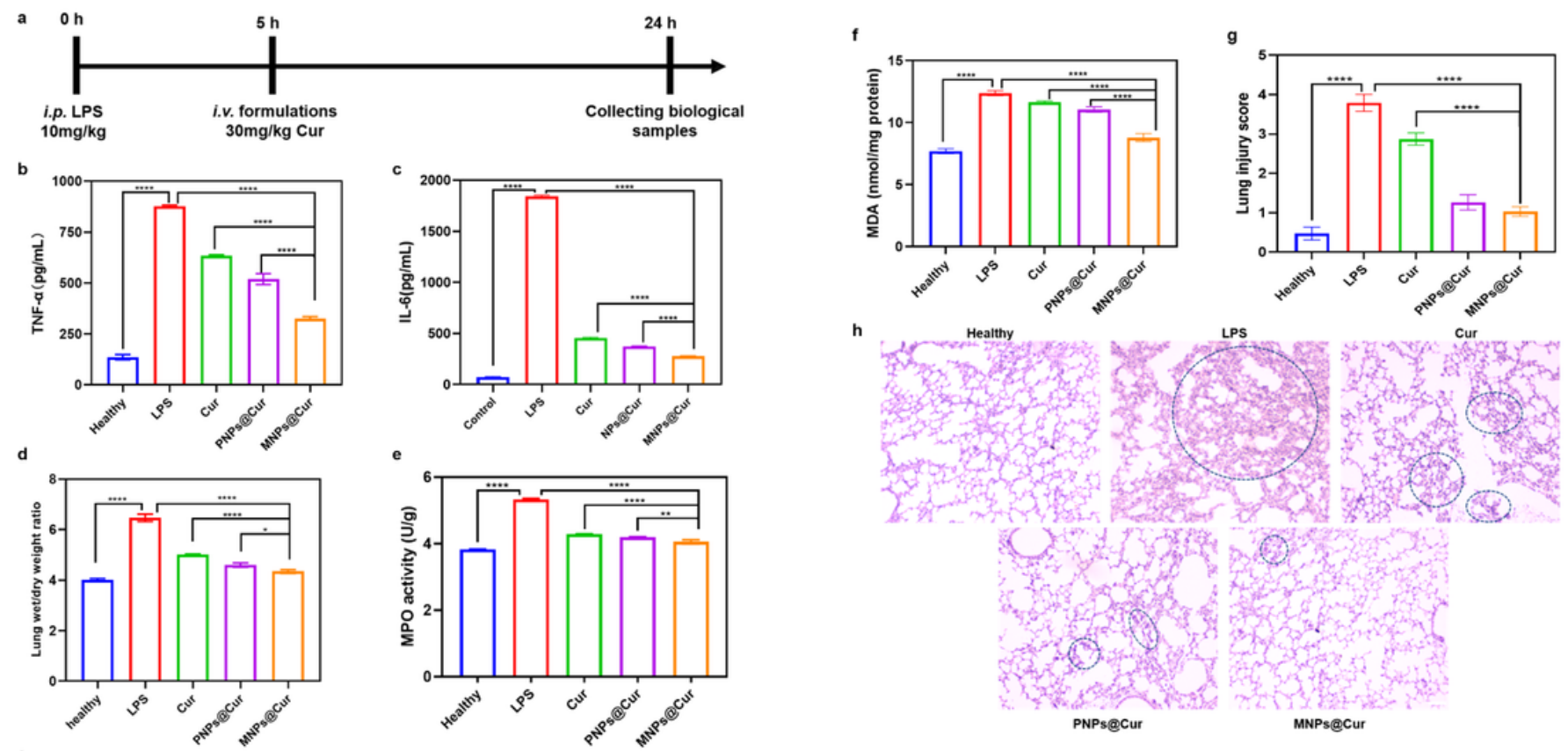

\section{Figure 8}

Targeted delivery of MNPs@Cur inhibits cytokine storm to improve lung inflammation. a The schedule of experiments for ALI model studies. ALI mouse models were induced by intraperitoneal injection of LPS (10 mg/kg). 5 hours after LPS administration, mice were injected intravenously with saline, free Cur, PNPs@Cur, and MNPs@Cur and 19 hours after drug administration, lungs were collected for treatment analysis. b, c The expression levels of inflammatory factors TNF-a, IL-6 in the serum of mice after various treatments. $d$ The wet-to-dry ratios of the lungs by different treatments indicated in the diagram. $e, f$ The levels of MPO and MDA in lung tissue homogenates after various treatments, as shown. g,h Semiquantitative analysis of lung damage scores and H\&E-stained lung tissue sections were imaged. The blue circles indicate neutrophil infiltration. Scale bar: $100 \mu \mathrm{m}$. Statistical significance was calculated by oneway ANOVA $(n=3-5)$. P-value: ${ }^{*} P<0.05,{ }^{\star} * P<0.01$ and ${ }^{\star * \star} P<0.001$. Data are means $\pm S D$ 


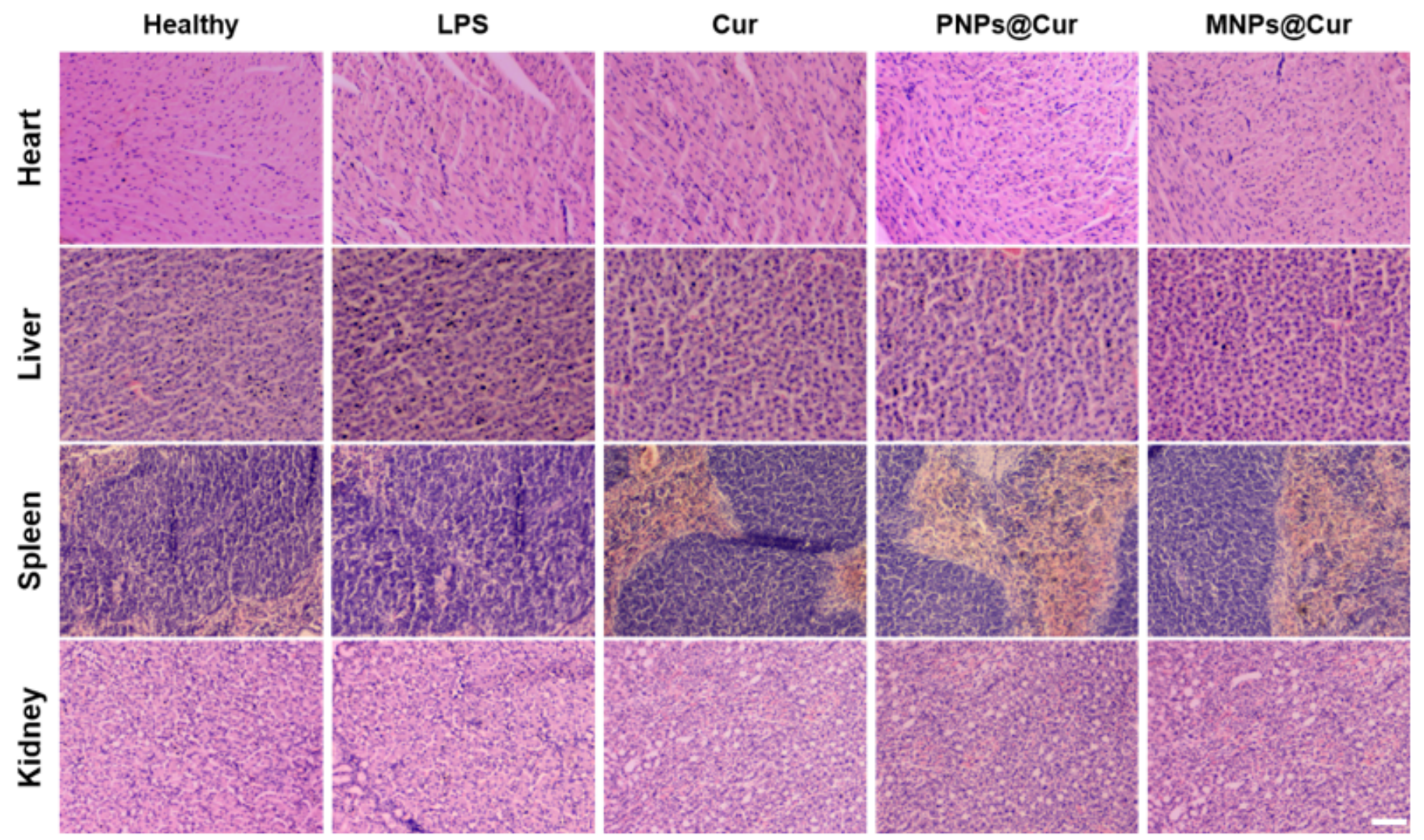

\section{Figure 9}

Samples were collected from the heart, liver, kidney and spleen, sectioned and stained (H\&E) for histological analysis (scale bar $=100 \mu \mathrm{m}$ ). There were no signs of tissue damage or disorganization of tissue structure found in any of the tissues

\section{Supplementary Files}

This is a list of supplementary files associated with this preprint. Click to download.

- GraphicalAbstract.docx 